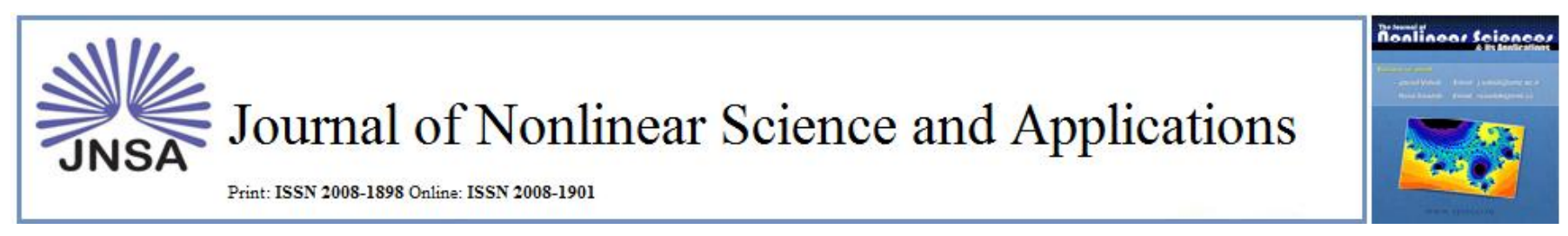

\title{
On fuzzy order relations
}

\author{
Ismat Beg* \\ Lahore University of Management Sciences \& University of Central Punjab, Lahore, Pakistan \\ Dedicated to George A Anastassiou on the occasion of his sixtieth birthday
}

\begin{abstract}
In this review article we present results regarding the fuzzy order relations. The concept of fuzzy order was introduced by generalizing the notion of reflexivity, antisymmetric and transitivity.

Keywords: Ordered set; fuzzy ordered set; Zorn's lemma; fixed point; selection, extension; variational principle; multivalued mapping; fuzzy metric spaces; fuzzy Riesz spaces; fuzzy positive linear operator; Hahn-Banach theorem.

2010 MSC: Primary 46S40; Secondary 03E72; 47H10; 47S40; 06A06; 46A40; 06F20; 49J40; 47A20.
\end{abstract}

\section{INTRODUCTION}

The notion of fuzzy set theory was initiated by Zadeh in 1965 in his seminal paper [90]. This notion tries to show that an object corresponds more or less to the particular category we want to assimilate it to. The notion of a fuzzy set is completely non-statistical in nature and the concept of fuzzy set provides a natural way of dealing with problems in which the source of imprecision is the absence of sharply defined criteria of class membership rather than the presence of random variables. In fact the idea of describing all shades of reality was for long the obsession of some logicians [71] [83]. During last four decades the fuzzy set theory has rapidly developed into an area which scientifically as well as from the application point of view, is recognized as a very valuable contribution to the existing knowledge (see [72] [70] [94] 64] [43] [14] [15] [16] [17] [18 [19] 21] [22 [44 [65] ). Preference ordering have been the object of extensive study in econometrics and other fields [2] [3] [29] [41] [76] [17, [20] 24] [23]. There exists a wide variety of techniques for dealing with problems involving equivalence, similarity, clustering preference etc. The study of fuzzy relations was also started by Zadeh [91] in 1971. In that celebrated paper he introduced the concept of fuzzy relation, defined the notion of equivalence, and gave the concept of fuzzy ordering. The concept of fuzzy order was introduced by generalizing the notion of reflexivity, antisymmetric and transitivity, there by facilitating the derivation of known results in various areas and stimulating the discovery of new ones. Fuzzy ordering have

\footnotetext{
* Corresponding author

Email address: ibeg@lums.edu.pk (Ismat Beg)
} 
broad utility. They can be applied, for example, when expressing our preferences with a set of alternatives. Compared with crisp ordering, they have greater expressive power. They allow us to express not only that we prefer on alternative to another, but also the strength of this preference. In this review article we present results regarding the fuzzy order relations recently proved by the author in papers [5] [6] [7] [8] [9] [10] [11] [13] 25] [26] [27. In section 1, we present basic facts about ordered sets. In section 2, we prove fuzzy Zorn's lemma using fuzzy axiom of choice due to Chapin [35]. Section 3, deals with existence of fixed points of single/multi-valued mappings and obtains fuzzy analogue of several fundamental fixed point theorems. The aim of section 4, is to prove the existence of a fuzzy order preserving selectors for fuzzy multifunctions under suitable conditions. A fixed point theorem for fuzzy order preserving fuzzy multifunctions is also proved. In section 5, we further weaken the fuzzy transitivity condition without invoking any topological assumptions. A necessary and sufficient condition has been established to completely characterize the existence of maximal elements for general irreflexive nontransitive fuzzy relations. Section 6 is devoted to elucidate the connection between fixed point theorem and the basic completeness technique in fuzzy metric spaces. We show that fuzzy Ekeland theorem is a consequence of Beg ([9], Theorem 7) and it further characterizes complete fuzzy metric spaces. We also obtained a characterization in terms of existence of fixed points in fuzzy metric spaces. In section 7 , we study the extension of fuzzy positive linear operators defined on a majorizing vector subspace of a fuzzy Riesz space with values in a complete fuzzy Riesz space. The fuzzy analogue of the most general form of the famous Hahn-Banach theorem is also proved.

\section{ORDERED SETS}

There are two types of relations which often arise in mathematics: order relations and equivalence relations. An order relation is a generalization of both set inclusion and the order relation on real line.

A relation $R$, defined for some pairs $x, y$ of elements of a set $X$, is called an order relation in $X$ if it satisfies:

(i) $x R x$ for any $x \in X$ (reflexivity);

(ii) $x R y$ and $y R z$ imply $x R z$ (transitivity);

(iii) $x R y$ and $y R x$ imply $x=y$ (antisymmetric).

A set $X$ in which an order relation has been given is called an ordered set (semi-ordered set or partially ordered set).

If $Y$ is a subset of an ordered set $X$, then the relation $R$, considered for elements $x, y$ in $Y$, is an order relation in $Y$ and is called induced order relation. A relation $R$ which only satisfies (i) and (ii) is called a pre-order relation and a set in which a pre-order relation has been given is called a pre-ordered set.

Let $Y$ be a pre-ordered set with the pre-order relation $P$. The relation $\bar{\sim}$, defined by the condition $x \bar{\sim} y$ $\Longleftrightarrow " x P y$ and $y P x "$, is an equivalence relation in $Y$.

The symbol $\leq$ is often used for an order relation, i.e., we write $x \leq y$ instead of $x R y$ and read as $x$ is smaller or equal (or less preferred) to $y$. The notation $x<y$ is used for $x \leq y$ and $x \neq y$.

If for a given pair of $x, y$ in an ordered set $X$, the relation $x \leq y$ does not hold we write $x \not \leq y$. If $x \not \leq y$ and $y \not \leq x$, then the elements $x, y$ are called incomparable.

An ordered set $X$ is called totally ordered set if it has no incomparable elements.

Let $X$ be an ordered set. A subset $Y$ of $X$ is said to be bounded from below if there exists an element $y$ in $X$ such that $y \leq x$ for any $x$ in $Y$. The element $y$ is called a lower bound for $Y$. If there exists a lower bound $y$ for the set $Y$, which is in $Y$, then $y$ is called the smallest element of $Y$.

A subset $Z$ of $X$ is said to be bounded from above if there exists an element $z$ in $X$ such that $x \leq z$ for any $x$ in $Z$. The element $z$ is called an upper bound for $Z$. If there exists an upper bound $z$ for the set $Z$, which is in $Z$, then $z$ is called the greatest element of $Z$.

A subset of $X$, which is both bounded from below and above is called bounded.

If $a$ and $b$ are elements of $X$, such that $a \leq b$, then the set $[a, b]=\{x \in X ; a \leq x \leq b\}$ is called an interval.

A subset $Y$ of $X$ has a greatest lower bound if it is bounded from below and if the set of its lower bounds has a greatest element $a$. The element $a$ is called the greatest lower bound of $Y$ and it is denoted by Inf or 
$g l b Y$. The $g l b$ if it exists is unique.

A subset $Z$ of $X$ is said to has a least upper bound if it is bounded from above and if the set of its upper bounds has a smallest element $b$. The element $b$ is called the least upper bound of $Z$ and it is denoted by sup or $l u b Z$. The $l u b$ if it exists is unique.

We write $x \vee y$ for supremum and $x \wedge y$ for the infimum, of the two point set $\{\mathrm{x}, \mathrm{y}\}$. For total orders, $x \vee y=\max \{x, y\}$ and $x \wedge y=\min \{x, y\}$. A lattice is an ordered set in which every pair of elements has a supremum and infimum. It is easy to show (by induction) that every finite set in a lattice has a supremum and an infimum.

Zermelo-Frankel (ZF) set theory has eight axioms .There is also a ninth axiom, the axiom of choice: the Cartesian product of a nonempty set of nonempty sets is itself a nonempty set. ZF set theory together with this axiom is referred as ZFC set theory. The ninth axiom, the axiom of choice is a seemingly innocuous set theoretic axiom with much hidden power. This axiom is both consistent with and independent of ZF set theory proper. Even though the axiom of choice has some unpleasant consequences but dropping the axiom of choice has also more devastating side effects. The biggest problem is that some of the most useful tools of analysis ( like Tychonoff Product Theorem, most proofs of Hahn-Banach Extension Theorem, existence of non-Lebesgue measurable sets of real numbers, etc.) would be thrown out with it and life would be nasty and brutish.

For more detailed study we refer to [56] [57] [58, [73] [52] [42].

Let $X$ be an ordered set. An element $a$ in $X$ is said to be minimal if $x \leq a$ implies $x=a$. An element $b$ in $X$ is said to be maximal if $b \leq x$ implies $x=b$.

Zorn's Lemma [95]: Let $X$ be an ordered set, such that any totally ordered subset of $X$ has an upper bound. Then for any element $x_{0} \in X$ there exists a maximal element $y$ in $X$, such that $x_{0} \leq y$.

One of the most useful proposition equivalent to axiom of choice is Zorn's lemma. That is, Zorn's lemma is a theorem if the axiom of choice is assumed, but if Zorn's lemma is taken as an axiom, then axiom of choice becomes a theorem. Zorn's lemma is a very powerful tool and is used to prove a number of results in mathematics and economics e.g., that vector spaces possess Hamel basis, any preorder have a compatible extension to a total preorder, Knaster-Tarski fixed point theorem, key results in revealed preference etc. For detail see [81] [82] [85] [2].

\section{FUZZY ZORN'S LEMMA}

Zorn's lemma is one of the most famous and useful result in mathematics. Chapin Jr. 35] [36] studied the basic logical axioms of fuzzy set theory. In this section we prove fuzzy Zorn's lemma using fuzzy axiom of choice due to Chapin Jr [35].

Let $X$ be a space of objects, with generic elements of $X$ denoted by $x$. A fuzzy subset $A$ of $X$ is characterized by a membership function $a$ which associates with each element in $X$ a real number in the interval $[0,1]$..

Definition 2.1. A fuzzy preorder $\mu$ on $X$ is a fuzzy subset of $X \times X$ such that the following conditions are satisfied:

(i) for all $x \in X, \mu(x, x)=1$,

(ii) for all $(x, y, z) \in X^{3}$,

$$
\mu(x, z) \geq \underset{y}{\operatorname{Max}}[\operatorname{Min}\{\mu(x, y), \mu(y, z)\}] .
$$

A nonempty set $X$ with fuzzy preorder $\mu$ defined on it, is called fuzzy preordered set and we denote it by $(X, \mu)$ or just by $X$ for simplicity sake when there is no confusion. A fuzzy preordered set is called fuzzy ordered set if:

(iii) $\mu(x, y)+\mu(y, x)>1$ implies $x=y$

Remark 2.2. Let $X$ be a fuzzy preordered set. The fuzzy preorder $\mu$ is said to be linear (total) if for all 
$x \neq y$, we have $\mu(x, y) \neq \mu(y, x)$. A fuzzy subset on which fuzzy preorder is linear is called a fuzzy chain. For a subset $A \subset X$, an upper bound (strict upper bound) is an element $x \in X$ satisfying $\mu(y, x) \geq \mu(x, y)$ $(\mu(y, x)>\mu(x, y))$ for all $y$ in $A$. An element $x$ is a maximal element of $X$ if $\mu(x, y) \geq \mu(y, x)$ for some $y \in X$, then $\mu(x, y)+\mu(y, x)>1$. The set of all maximal elements of $X$ will be denoted by $\sup (X)$. Minimal elements are defined analogously. A greatest element of $A$ is an $x \in A$ satisfying $\mu(y, x) \geq \mu(x, y)$ for all $y \in A$. Least elements are defined in the obvious fashion.

For more details see Billot [29], Beg and Islam [26], Novak [75], Li and Yen [70].

Let $A$ and $B$ be two fuzzy subsets of $X$, then $(a-b)(x)=\min \{a(x), 1-b(x)\}, \quad(A \cup B)(x)=$ $\max \{a(x), b(x)\}$ and $(A \cap B)(x)=\min \{a(x), b(x)\}$. A fuzzy set is empty if it is the constant zero function. Using Fuzzy Axiom of Choice (Chapin [35], Ax. 14 ), we choose a function $f$ that assigns to every bounded fuzzy chain $C$ a strict upper bound $f(C)$.

For further details and basic logical axioms of fuzzy set theory we refer to Zadeh [90] [91, Brown [32], Chapin [35] [36], [78] [79] [55, Zimmermann [94] and Dubois and Prade [43].

Definition 2.3. If $C$ is a fuzzy chain in $X$ and $x \in C$, then we define the fuzzy subset $P(C, x)$ of $C$ by $P(C, x)(y)=\mu(y, x)-\mu(x, y)$. A fuzzy subset of a fuzzy chain $C$ that has the form $P(C, x)$ is called an initial segment in $\mathrm{C}$.

Definition 2.4. A fuzzy subset $A$ of a fuzzy chain $C$ is called conforming if the following two conditions hold:

(iv) Every non-empty fuzzy subset of A has least element.

(v) For every $x$ in $A, x=f(P(A, x))$.

Proposition 2.5. Let $A$ and $B$ be conforming subsets of a fuzzy chain $C$ and $A \neq B$, then one of these two sets is an initial segment of the other.

Proof. We may assume $A-B \neq \phi$, i.e., $\min \{a(x), 1-b(x)\} \neq 0$ for some $x \in A$. Define $x_{0}$ to be the least element of $A-B$. Thus $\mu\left(x_{0}, y\right) \geq \mu\left(y, x_{0}\right)$ if $\min \{a(y), 1-b(y)\} \neq 0$. Therefore, if $\mu\left(x_{0}, y\right)<\mu\left(y, x_{0}\right)$, then either $a(y)=0$ or $b(y)=1$. We claim that $P\left(A, x_{0}\right)=B$. For this we show that:( i) $P\left(A, x_{0}\right) \subseteq B$ and (ii) $B-P\left(A, x_{0}\right)=\phi$.

(i) If $y \in P\left(A, x_{0}\right)$, then $P\left(A, x_{0}\right)>0$. Thus $\mu\left(y, x_{0}\right)-\mu\left(x_{0}, y\right)>0$. It implies that $\mu\left(x_{0}, y\right)<\mu\left(y, x_{0}\right)$. Moreover, $y \in A$ by definition 2.4. Thus $b(y)=1$. It further implies that $P\left(A, x_{0}\right) \leq b(y)$. Therefore, $P\left(A, x_{0}\right) \subseteq B$.

(ii) Assume that $B-P\left(A, x_{0}\right) \neq \phi$ and let $y_{0}$ be the least element of $B-P\left(A, x_{0}\right)$, i.e., $\min \left\{b\left(y_{0}\right), 1-\right.$ $\left.P\left(A, x_{0}\right)\left(y_{0}\right)\right\} \neq 0$ and $\mu\left(y, y_{0}\right) \leq \mu\left(y_{0}, y\right)$ for all $y \in B-P\left(A, x_{0}\right)=B-\left\{y \in A: \mu\left(y, x_{0}\right)>\mu\left(x_{0}, y\right)\right\}$. It implies that $P\left(B, y_{0}\right) \subseteq P\left(A, x_{0}\right)$. Given any element $u \in P\left(B, y_{0}\right)$ and any element $v \in A$ such that $\mu(v, u)>\mu(u, v)$. Obviously $v \in P\left(A, x_{0}\right) \subseteq B$. Since $\mu\left(u, y_{0}\right)>\mu\left(y_{0}, u\right)$ then $\mu\left(v, y_{0}\right)>\mu\left(y_{0}, v\right)$. It further implies that $v \in P\left(B, y_{0}\right)$. Therefore, if $z_{0}$ is the least element of $A-P\left(B, y_{0}\right)$, we have $P\left(A, z_{0}\right)=P\left(B, y_{0}\right)$. Note that $\mu\left(z_{0}, x_{0}\right) \geq \mu\left(x_{0}, z_{0}\right)$. But since $z=f\left(P\left(A, z_{0}\right)\right)=f\left(P\left(B, y_{0}\right)\right)=y_{0}$ and since $y_{0} \in B$, we can not have $z_{0}=x_{0}$. Therefore $\mu\left(z_{0}, x_{0}\right)>\mu\left(x_{0}, z_{0}\right)$ and we conclude $y_{0}=z_{0} \in P\left(A, x_{0}\right)$, contradicting the choice of $y_{0}$.

Remark 2.6. If $A$ is a conforming subset of $X$ and $x \in A$, then whenever $\mu(y, x)>\mu(x, y)$, either $y \in A$ or $y$ does not belong to any conforming set. Therefore, it follows that the union $\Omega$ of all conforming subset of $X$ is conforming.

Theorem 2.7. Fuzzy Zorn's lemma: Let $X$ be a fuzzy ordered set with fuzzy order $R$. If every fuzzy chain in $X$ has an upper bound then $X$ has a maximal element.

Proof. Suppose that $X$ has no maximal element. If $C$ is a chain in $X$, then by choosing an upper bound $u$ of $C$ and then choosing an element $x, \mu(u, x)>\mu(x, u)$. We can obtain an element $x$ in $X$ such that $\mu(y, x)>\mu(x, y)$ for every $y$ in $C$. Then $x$ will be a strict upper bound of $C$. If $x=f(\Omega)$ then the set $\Omega \cup[x\}$ is conforming by Remark 2.6. Therefore $x \in \Omega$, contradicting the fact that $x$ is a strict upper bound of $\Omega$.

Remark 2.8. Proposition 2.5 and theorem 2.7 are from Beg [7]. 


\section{FIXED POINT}

Fixed point theorems are fundamental tools for solving functional equations. Recently Heilpern [54], Kaleva [61], Bose and Sahani [31, Park and Jeong [77, Lee and Cho [69], Beg [5] and many other authors have proved fixed point theorems in fuzzy setting, specially for fuzzy metric spaces. Zadeh [91] and Negoita and Ralescu [74] have introduced the notion of fuzzy order and similarity in their papers, which was subsequently further developed and refined by Venugopalan [87, Orlovsky [76], Billot [29], Kundu [68] and Beg and Islam [25] [26] 27]. In this section, we present results on existence of fixed points of single/multi-valued mappings and obtain fuzzy analogue of several fundamental fixed point theorems.

Definition 3.1. A set $X$ is well fuzzy preordered by the linear fuzzy preordered $\mu$ if every nonempty subset of $X$ has a least element.

Definition 3.2. A fuzzy preordered set $X$ is called $A$-inductive if and only if every nonempty well fuzzy preordered subset of it has a least upper bound (abbreviated as lub).

Definition 3.3. Let $X$ be a nonempty fuzzy preordered set. A mapping $f: X \rightarrow X$ is called expansive if $\mu(x, f(x)) \geq \mu(f(x), x)$ for every $x \in X$.

Definition 3.4. Let $f: X \longrightarrow X$, then a point $x \in X$ is called a fixed point of $f$ if $f(x)=x$.

Theorem 3.5. Let $X$ be a nonempty A-inductive fuzzy ordered set and $f: X \rightarrow X$ be an expansive mapping then $f$ has a fixed point.

Proof. Assume that $f$ has no fixed point. Since fuzzy order $\mu$ is linear therefore for every $x \in X . \mu(x, f(x))>$ $\mu(f(x), x)$. Let $z$ be an arbitrary element of $X$. For every ordinal $p$ we define:

$$
f^{p}(z)=\left\{\begin{array}{l}
z \text { if } \mathrm{p}=0, \\
f\left(f^{p-1}(z)\right) \text { if } \mathrm{p} \text { is a nonzero nonlimit ordinal } \\
\operatorname{lub} f^{i}(z) \text { if } \mathrm{p} \text { is a limit ordinal. }
\end{array}\right.
$$

Since $X$ is A-inductive, it follows that for every ordinal $p, f^{p}(z) \in X$. Moreover, for every ordinal $p$ and $q$ if $p \neq q$ then $f^{p}(z) \neq f^{q}(z)$. Now, to every $x \in X$, assign an ordinal $F(x)$ as follows:

$$
F(x)=\left\{\begin{array}{l}
p \text { if } x=f^{p}(z) \\
o \text { otherwise }
\end{array}\right.
$$

Obviously, $F$ is an ordinal valued function, whose domain is $X$. Since $X$ is a set, from the ZF Axiom of Substitution (Kelley [63, page 261) it follows that the range of $F$ is also a set. But range of $F$ is collection of all ordinals, which is not a set. Hence a contradiction. Thus our assumption is wrong and $f$ has a fixed point.

Remark 3.6. Let $X$ be a fuzzy preordered set. Define the fuzzy semilitude subrelation $\sim$ in $X$ by : $x \sim y$ if $\mu(x, y)+\mu(y, x)>1$. The relation $\sim$ between the different semilitude classes is necessarily antisymmetric (Negoita and Ralescu [74]). Semilitude classes are not necessarily disjoint. It is obvious that there exists a fuzzy order between semilitude classes.

Definition 3.7. A nonempty subset $E$ of a fuzzy preordered set $X$ is called fuzzy order extremal provided $x \sim y$ for all $x, y \in E$; and $x \in E$ whenever $x \in X$ and $\mu(y, x)>\mu(x, y)$ for some $y \in E$.

Remark 3.8. A fuzzy order extremal subset $E$ is always a chain, has a least upper bound and it consists of maximal elements of $X$.

Remark 3.9. A singleton set $E=\{x\}$ is fuzzy order extremal if and only if $x$ is a unique least upper bound of $\{y: y \in X$ and $\mu(x, y) \geq \mu(y, x)\}$.

Lemma 3.10. Let $X$ be a nonempty A-inductive fuzzy preordered set and let $f: X \rightarrow X$ be an expansive 
mapping. Then there exists a fuzzy order extremal f-invariant set $W$ in $\sup (X)$.

Proof. Let $Y=\left\{D_{x}: D_{x}=\{y \in X: y \sim x\}\right\}_{x \in X}$. Define the fuzzy order $\lambda$ in $Y$ by: $\lambda\left(D_{x}, D_{y}\right) \geq \lambda\left(D_{y}, D_{x}\right)$ if $\mu(x, y) \geq \mu(y, x)$ (see Remark 3.6). Since $X$ is A-inductive fuzzy preordered set, it follows that $Y$ is A-inductive fuzzy ordered set. The fuzzy ordered set $Y$ has a maximal element $W$. Otherwise, for each $D \in Y$, there exists a semilitude class $h(D) \in Y$ such that $\lambda(D, h(D)) \geq \lambda(h(D), D)$ and $h(D) \neq D$. Hence Theorem 3.5 is contradicted by the mapping $h: Y \rightarrow Y$. Clearly, the maximal element $W \in Y$, is order extremal and $W \subset \sup (X)$. Moreover, if $x \in W$, then $\mu(x, f(x)) \geq \mu(f(x), x)$. It further implies that $f(x) \in W$. Thus $W$ is f-invariant.

Definition 3.11. An A-inductive fuzzy preordered set $X$ is said to have normal fuzzy order structure if each fuzzy order extremal subset of $X$ is a singleton.

Theorem 3.12. Let $X$ be a fuzzy preordered set with normal fuzzy order structure and $f: X \rightarrow X$ be an expansive mapping. Then $f$ has a maximal fixed point.

Proof. Let $X$ be a fuzzy preordered set with normal fuzzy ordered structure. If $\mu(x, f(x)) \geq \mu(f(x), x)$ for every $x \in X$ then the fuzzy ordered extremal set $W \subset \sup (X)$, constructed in Lemma 3.10 is a singleton, say $W=\{w\}$. Since $W$ is f-invariant, therefore $f(w)=w$. Maximality of $W$ in $Y$ implies that $w$ is a maximal fixed point.

Theorem 3.13. Let $X$ be a fuzzy ordered set and $X_{0}$ be an $A$-inductive fuzzy subset of $X$. Let $f: X_{0} \rightarrow X_{0}$ and $g: X \rightarrow X$ be expansive mappings. If $g(f i x f) \subset X_{0}$ then $f$ and $g$ have a common fixed point.

Proof. By theorem 3.5, there exists an expansive mapping $h: X_{0} \rightarrow f i x(f)$. Then the mapping goh is expansive and $g o h\left(X_{0}\right) \subseteq g(f i x(f)) \subseteq X_{0}$. Theorem 3.5, further implies that goh has a fixed point $x^{*}$. Because $h$ is expansive therefore

$$
\mu\left(x^{*}, h\left(x^{*}\right)\right) \geq \mu\left(h\left(x^{*}\right), x^{*}\right) .
$$

Also $g$ is expansive, thus

$$
\mu\left(h\left(x^{*}\right), g\left(h\left(x^{*}\right)\right)\right) \geq \mu\left(g\left(h\left(x^{*}\right)\right), h\left(x^{*}\right)\right) .
$$

Since $x^{*}=g o h\left(x^{*}\right)$, therefore it gives

$$
\mu\left(x^{*}, h\left(x^{*}\right)\right) \geq \mu\left(h\left(x^{*}\right), x^{*}\right) \text { and } \mu\left(h\left(x^{*}\right), x^{*}\right) \geq \mu\left(x^{*}, h\left(x^{*}\right)\right) .
$$

It further implies $\mu\left(x^{*}, h\left(x^{*}\right)\right)=\mu\left(h\left(x^{*}\right), x^{*}\right)$. Linearity of fuzzy order $\mu$ now implies $x^{*}=h\left(x^{*}\right)$. Thus $g\left(x^{*}\right)=g\left(h\left(x^{*}\right)\right)=g o h\left(x^{*}\right)=x^{*}$, so $x^{*}$ is a fixed point of $g$.

Since $f o h=h, x^{*}$ is a fixed point of $f o h$. Repeating the above argument yields $x^{*}=f\left(x^{*}\right)$. Hence $x^{*}$ is a common fixed point of $f$ and $g$.

Definition 3.14. A mapping $f: X \rightarrow X$ is called fuzzy monotone if $\mu(y, x)) \geq \mu(x, y)$ implies $\mu(f(y), f(x) \geq \mu(f(x), f(y))$.

Theorem 3.15. Let $X$ be a fuzzy ordered set with the property that every fuzzy chain in $X$ has a supremum. Let $f: X \rightarrow X$ be a fuzzy monotone map and assume that there exists some $a \in X$ with $\mu(a, f(a) \geq$ $\mu(f(a), a)$. Then the set of fixed points of $f$ is nonempty and has a maximal fixed point.

Proof . Consider the fuzzy ordered subset $P=\{x \in X: \mu(x, f(x)) \geq \mu(f(x), x)\}$. Since $a \in P$, therefore $P$ is nonempty. Let $C$ be a chain in $P$ and $b$ be its supremum in $X$. Then $\mu(c, b) \geq \mu(b, c)$ for every $c \in C$. Thus $\mu(f(c), f(b)) \geq \mu(f(b), f(c))$. As $\mu(c, f(c)) \geq \mu(f(c), c)$, therefore $\mu(c, f(b)) \geq \mu(f(b), c)$ for $c \in P$. It follows that $f(b)$ is an upper bound of $C$. Since $b$ is supremum of $C$ and $f(b)$ is an upper bound for $C$, we have $\mu(b, f(b)) \geq \mu(f(b), b)$. Therefore $b \in P$. Thus supremum of any chain $P$ belongs to $P$. Fuzzy Zorn's Lemma further implies that $P$ has a maximal element, $x_{0}$ (say). Since $x_{0} \in P, \mu\left(x_{0}, f\left(x_{0}\right)\right) \geq \mu\left(f\left(x_{0}\right), x_{0}\right)$. 
As $f$ is monotone, $\mu\left(f\left(x_{0}\right), f\left(f\left(x_{0}\right)\right)\right) \geq \mu\left(f\left(f\left(x_{0}\right)\right), f\left(x_{0}\right)\right)$. It further implies that $f\left(x_{0}\right)$ belong to $P$. Since $x_{0}$ is a maximal element of $P$, we see that $x_{0}=f\left(x_{0}\right)$.

Furthermore, if $y$ is another fixed point of $f$, then $y \in P$. This shows that $x_{0}$ is a maximal fixed point of $f$.

Definition 3.16. A map $f: X \rightarrow X$ is said to be fuzzy order continuous if for each countable fuzzy chain $\left\{c_{i}\right\}$ having a supremum, $f\left(\sup \left\{c_{i}\right\}\right)=\sup \left(\left\{f\left(c_{i}\right)\right\}\right)$.

A fuzzy order continuous map is necessarily monotone; for if $\mu(x, y) \geq \mu(y, x)$ then $y=\sup \{x, y\}$, so by continuity, $f(y)=\sup \{f(x), f(y)\}$. Therefore

$$
\mu(f(x), f(y)) \geq \mu(f(y), f(x)) .
$$

Theorem 3.17. Let $X$ be a fuzzy ordered set and $f: X \rightarrow X$ be fuzzy order continuous map. Assume that there is a $b \in X$ such that:

i) $\mu(b, f(b)) \geq \mu(f(b), b)$, and

ii) every countable fuzzy chain in $\{x: \mu(b, x) \geq \mu(x, b)\}$ has a supremum.

Then the point $c=\sup f^{n}(b)$ is a fixed point of $f$. Moreover the point $c$ is also the infimum of the set of fixed points of $f$ in $\{x: \mu(b, x) \geq \mu(x, b)\}$.

Proof. Because $\mu(b, f(b)) \geq \mu(f(b), b)$ and $f$ is monotone, we have

$$
\mu\left(f(b), f^{2}(b)\right) \geq \mu\left(f^{2}(b), f(b)\right),
$$

and inductively,

$$
\mu\left(f^{n}(b), f^{n+1}(b)\right) \geq \mu\left(f^{n+1}(b), f^{n}(b)\right),
$$

for each $n \geq 1$. Thus $\left\{f^{n}(b): n \geq 1\right\}$ is a chain in $\{x: x \geq b\}$; so $c=\sup _{n} f^{n}(b)$ exists.

Since $f$ is continuous,

$$
f(c)=f\left(\sup _{n} f^{n}(b)\right)=\sup _{n} f\left(f^{n}(b)\right)=\sup _{n} f^{n+1}(b)=c .
$$

Hence $c$ is a fixed point of $f$. Let $e$ be another fixed point of $f$ in $\{x: \mu(b, x) \geq \mu(x, b)\}$, then $\mu(c, e) \geq$ $\mu(e, c)$. Indeed, since $\mu(b, e) \geq \mu(e, b)$, we have $\mu(f(b), e)=\mu(f(b), f(e)) \geq \mu(f(e), f(b))=\mu(e, f(b))$, and by induction, that $\mu\left(f^{n}(b), e\right) \geq \mu\left(e, f^{n}(b)\right)$ for every $n \geq 1$. Thus $e$ is an upper bound for $\left\{f^{n}(b): n \geq 1\right\}$, so $\mu(c, e) \geq \mu(e, c)$. Thus $c$ is the infimum of the set of fixed points of $f$ in $\{x: \mu(b, x) \geq \mu(x, b)\}$.

Next we prove some fixed point theorems for fuzzy multivalued mappings. First we state some definitions and notations.

Let $(X, d)$ be a metric linear space (i.e., a complex or real vector space ) and $A$ be a fuzzy set in $X$ characterized by a membership function $\alpha$. The $\lambda$-level set of $A$, denoted by $A_{\lambda}$, is defined by

$$
\begin{aligned}
& A_{\lambda}=\{x: \alpha(x) \geq \lambda\} \text { if } \lambda \in(0,1], \\
& A_{0}=\overline{\{x: \alpha(x)>0\}},
\end{aligned}
$$

where $\bar{B}$ denotes the closure of the (nonfuzzy) set $B$.

Definition 3.18. A fuzzy set $A$ in a metric linear space $X$ is said to be an approximate quantity if and only if $\mathrm{A}_{\lambda}$ is compact and convex in $X$ for each $\lambda \in[0,1]$ and $\sup _{x \in X} \alpha(x)=1$.

Let $W(X)$ be a collection of all approximate quantities in $X$.

Definition 3.19. Let $A, B \in W(X)$. Then $A$ is said to be more accurate than $B$ (or $B$ includes $A$ ) denoted 
by $A \subset B$ if and only if $\alpha(x) \leq \beta(x)$ for each $x \in X$, where $\beta$ is the membership function of $B$. The relation $\subset$ induces an order on $W(X)$.

Let $X$ be a nonempty fuzzy ordered set and $T$ be a fuzzy multivalued mapping with domain in $X$ and range in the family of all fuzzy subsets of $X$ i.e., $T: X \rightarrow[0,1]^{X}$. We assume that $T$ is nonempty valued, meaning that $T(x) \neq \phi$ for all $x \in X$. $T(a) \geq a$ if and only if for all $x \in T(a), \mu(a, x) \geq \mu(x, a)$. A point $x \in X$ is called a fixed point of $T$ if $\{x\} \subset T(x)$.

Lemma 3.20. Let $X$ be a fuzzy ordered set. Let $T: X \rightarrow[0,1]^{X}$ be a nonempty fuzzy multivalued mapping and there exists some $a \in X$ such that $T(a) \geq a$. Let $P=\{x \in X: T(x) \geq x\}$. Assume that every nonempty T-invariant subset $A$ of $P$ has a maximal point. Then $T$ has a fixed point.

Proof. Let $a \in A$ be a maximal point of $A$. Since $a \in A \subset P$. Therefore for all $b \in T(a), \mu(a, b) \geq \mu(b, a)$. As $T(a) \subset T(A) \subset A$, maximality of $a$ in $A$ ensures that for all $b \in T(a), a=b$, proving that $\{a\}=T(a)$.

Definition 3.21. Let $X$ be a fuzzy ordered set and $T: X \rightarrow[0,1]^{X}$. The fuzzy multivalued mapping $T$ is called nondecreasing if $\mu(a, b) \geq \mu(b, a)$ and $a \neq b$ imply that for all $x \in T(a)$, for all $y \in T(b), \mu(x, y) \geq$ $\mu(y, x)$.

Lemma 3.22. Let $X$ be a fuzzy ordered set. Let $T: X \rightarrow[0,1]^{X}$ be a nonempty nondecreasing fuzzy multivalued mapping and there exists some $a \in X$ such that $T(a) \geq a$. Let $P=\{x \in X: T(x) \geq x\}$. Then $T(P) \subset P$.

Proof. Let $y \in T(P)$. Then there exists $x \in P$, such that $y \in T(x)$. As $x \in P$, therefore for all $z \in T(x)$, $\mu(x, z) \geq \mu(z, x)$. In particular,$\mu(x, y) \geq \mu(y, x)$. If $y=x$, the result follows. If $y \neq x$, then as $T$ is nondecreasing, therefore for all $z_{1} \in T(x)$ and $z_{2} \in T(y), \mu\left(z_{1}, z_{2}\right) \geq \mu\left(z_{2}, z_{1}\right)$. As $y \in T(x)$, we can conclude that for all $z_{2} \in T(y), \mu\left(y, z_{2}\right) \geq \mu\left(z_{2}, y\right)$. Hence $y \in P$.

Theorem 3.23. Let $X$ be a fuzzy ordered set in which every nonempty fuzzy chain has a supremum. Let $T: X \rightarrow[0,1]^{X}$ be a nonempty nondecreasing multivalued fuzzy mappings and there exits some $a \in X$ such that $T(a) \geq a$. Then there exits $x_{0} \in X$ such that $\left\{x_{0}\right\}=T\left(x_{0}\right)$.

Proof. Consider $P=\{x \in X: T(x) \geq x\}$. Since $T(a) \geq a, P$ is nonempty. Take a nonempty fuzzy chain $C$ in $P$. By hypothesis $C$ has a supremum $b$ in $X$, i.e., $\mu(c, b) \geq \mu(b, c)$ for all $c \in C$. If $b=c$ for some $c \in C$, then $b \in P$. Suppose now that $b \neq c$ for all $c \in C$. Since $T$ is nondecreasing, thus for all $y \in T(c)$ and for all $z \in T(b), \mu(y, z) \geq \mu(z, y)$. As $c \in C \subset P$, therefore $\mu(c, y) \geq \mu(y, c)$ for all $y \in T(c)$. It further implies that $\mu(c, z) \geq \mu(z, c)$ for all $z \in T(b)$. As $c$ is an arbitrary element of $C$, therefore every $z$ in $T(b)$ is a majorant of $C$ and $b$ is the smallest element of all majorants i.e., $\mu(b, z) \geq \mu(z, b)$ for all $z \in T(b)$. Thus $b \in P$. By fuzzy Zorn's Lemma, $P$ has a maximal element $x_{0}$. Lemma 3.22 implies that $T(P) \subset P$. Lemma 3.20 further implies that $\left\{x_{0}\right\}=T\left(x_{0}\right)$.

Remark 3.24. Theorem 3.23 is fuzzy multivalued version of the results of Knaster [67] and Tarski [86].

Now we prove a general principle on fuzzy ordered sets for use to prove a Caristi type fixed point theorem [33] for fuzzy multivalued mappings.

Let $X$ be a fuzzy ordered set. For $x \in X$, we denote $S(x)=\{y \in X: \mu(x, y) \geq \mu(y, x)\}$.

Definition 3.25. A sequence $\left\{x_{n}\right\}$ in a fuzzy ordered set $\mathrm{X}$ is said to be increasing, provided $\mu\left(x_{n}, x_{n+1}\right) \geq$ $\mu\left(x_{n+1}, x_{n}\right)$ for all $n$.

Theorem 3.26. Let $\psi: X \rightarrow R$ be a function which satisfies :

i) $\mu(x, y) \geq \mu(y, x)$ and $x \neq y$ implies $\psi(x) \leq \psi(y)$,

ii) for any increasing sequence $\left\{x_{n}\right\}$ in $X$ such that $\psi\left(x_{n}\right)<c<\infty$ for all $n$, there exists some $y \in X$ such that $\mu\left(x_{n}, y\right) \geq \mu\left(y, x_{n}\right)$ for all $n$, 
iii) for each $x \in X, \psi(S(x))$ is bounded.

Then for each $x \in X$, there exists $x^{\prime} \in S(x)$ such that $x^{\prime}$ is maximal i.e., $\left\{x^{/}\right\}=S\left(x^{\prime}\right)$.

Proof. For $a \in X$, let $p(a)=\sup \{\psi(b): b \in S(a)\}$. Assume the conclusion of the theorem fails for some $x \in$ $X$ and define by induction a sequence $\left\{x_{n}\right\}$ such that $x_{1}=x$ and $x_{n+1} \in S\left(x_{n}\right)$ satisfies $p\left(x_{n}\right) \leq \psi\left(x_{n+1}\right)+\frac{1}{n}$ for all $n$.

Since $\psi\left(x_{n+1}\right) \leq p\left(x_{n}\right)$ and $p\left(x_{n}\right) \neq+\infty$, it follows from (ii) that there exists some $y \in X$ such that $\mu\left(x_{n}, y\right) \geq \mu\left(y, x_{n}\right)$ for all $n$. Also by assumption $y$ is not maximal in $S(x)$, so there exists $u \in X$ such that $\mu(y, u) \geq \mu(u, y)$ and $\psi(y)<\psi(u)$. Since $\mu\left(x_{n}, u\right) \geq \mu\left(u, x_{n}\right), \psi(u) \leq p\left(x_{n}\right)$ for all $n$. Also $\mu\left(x_{n+1}, y\right) \geq \mu\left(y, x_{n+1}\right)$ thus $\psi\left(x_{n+1}\right) \leq \psi(y)$. Therefore

$$
\psi(u) \leq p\left(x_{n}\right) \leq \psi\left(x_{n+1}\right)+\frac{1}{n} \leq \psi(y)+\frac{1}{n}
$$

for all $n$. Hence $\psi(u) \leq \psi(y)$, a contradiction.

Theorem 3.27. Let $(X, d)$ be a complete metric linear space and $\varphi: X \rightarrow R$ be a bounded below lower semicontinuous function. Suppose $T: X \rightarrow W(X)$ is a fuzzy multivalued mapping such that for each $x \in X$, there exists $\{y\} \subset T(x)$ satisfying $d(x, y) \leq \varphi(x)-\varphi(y)$. Then there exists $x^{/} \in X$ such that $\left\{x^{/}\right\} \subset T\left(x^{\prime}\right)$. Proof. Set $\psi=-\varphi$ and for $x, y \in X$, say $\mu(x, y) \geq \mu(y, x)$ provided $d(x, y) \leq \varphi(x)-\varphi(y)$. Note that by assumption for every $u \in X$ there exists $v \in T(u)$ such that $\mu(u, v) \geq \mu(v, u)$. We must verify conditions (i), (ii), and (iii) of theorem 3.26. Condition (i) obviously holds. To see that (ii) holds, observe that if $\left\{x_{n}\right\}$ is any increasing sequence then $\left\{\varphi\left(x_{n}\right)\right\}$ is decreasing and bounded below, hence $\left\{\varphi\left(x_{n}\right)\right\}$ converges, say to $r \in \mathbb{R}$. It further implies that $\left\{x_{n}\right\}$ is a Cauchy sequence. Thus $\left\{x_{n}\right\}$ converges to a point $y \in X$ and since $\varphi$ is lower semicontinuous it follows that,

$$
d\left(x_{n}, y\right) \leq \varphi\left(x_{n}\right)-r \leq \varphi\left(x_{n}\right)-\varphi(y) .
$$

Thus $\mu\left(x_{n}, y\right) \geq \mu\left(y, x_{n}\right)$ for all $n$. Since (iii) follows from the fact that $\varphi$ is bounded below, we conclude [for each $u \in X$, put $f(u)=v$, then $\left.x^{\prime}=f\left(x^{\prime}\right)\right]$ that for each $x \in X$ there exists $x^{\prime}$ such that $\mu\left(x, x^{\prime}\right) \geq \mu\left(x^{\prime}, x\right)$ and $\left\{x^{/}\right\} \subset T\left(x^{/}\right)$.

Remark 3.28. Results presented in this section are mainly due to Beg [5] [8] [9] .

\section{SELECTION}

One of the most interesting and important problems in ordered set theory is the extension problem. Two ordered sets $X$ and $Y$ are given, together with a subset $A \subset X$, we would like to know whether every order preserving function $g: A \rightarrow Y$ can be extended to an order preserving function $f: X \rightarrow Y$. Sometimes there are additional requirements on $f$ e.g., for every $x \in X, f(x)$ must be an element of a pre-assigned subset of $Y$. This new problem is clearly more general than the extension problem and is called a selection problem. Even though there is a lot of work in the classical set theory on selection problems ( see; Knaster [67, Tarski [86, Davis [40], Birkhoff [30] and Smithson [84] ). The aim of this section is to prove the existence of a fuzzy order preserving selectors for fuzzy multifunctions under suitable conditions. A fixed point theorem for fuzzy order preserving fuzzy multifunctions is also proved.

Let $X$ be a fuzzy ordered set with a fuzzy order $R$ and $F: X \rightarrow[0,1]^{X} \backslash\{\phi\}$ be a fuzzy multifunction, that is, for $x \in X, F(x)$ is a nonempty fuzzy subset of $X$. If $F$ maps the points of its domain to singletons, then $F$ is said to be a single valued fuzzy function. No distinction will be made between a single valued fuzzy function and a fuzzy multifunction. The fuzzy multifunction $F$ is said to be fuzzy order preserving if and only if $x_{1}, x_{2} \in X$ and $y_{1} \in F\left(x_{1}\right), \mu\left(x_{1}, x_{2}\right) \geq \mu\left(x_{2}, x_{1}\right)$ implies that there is a $y_{2} \in F\left(x_{2}\right)$ such that $\mu\left(y_{1}, y_{2}\right) \geq \mu\left(y_{2}, y_{1}\right)$. A selector for $F$ is a fuzzy function $f: X \longrightarrow X$ such that $\{f(x)\} \subseteq F(x)$ for each $x \in X$. A point $x \in X$ is a fixed point of $F$, if $\{x\} \subseteq F(x)$.

Theorem 4.1. Let $X$ be a fuzzy ordered set and let $F$ be a fuzzy order preserving fuzzy multifunction on $X$. If $\sup F(x) \subseteq F(x)$ for all $x \in X$, then there is a fuzzy order preserving selector $f$ for $F$.

Proof. Let $f(x)=\sup F(x)$ for each $x \in X$. Then $f$ is a fuzzy order preserving selector for $F$. Indeed, let $\mu\left(x_{1}, x_{2}\right) \geq \mu\left(x_{2}, x_{1}\right)$. Since $\left\{f\left(x_{1}\right)\right\} \subseteq F\left(x_{1}\right)$ there is a $z \in F\left(x_{2}\right)$ such that $\mu\left(f\left(x_{1}\right), z\right) \geq \mu\left(z, f\left(x_{1}\right)\right)$. But 
$\mu\left(z, f\left(x_{2}\right)\right) \geq \mu\left(f\left(x_{2}\right), z\right)$. Hence $\mu\left(f\left(x_{1}\right), f\left(x_{2}\right)\right) \geq \mu\left(f\left(x_{2}\right), f\left(x_{1}\right)\right)$.

Theorem 4.2. Let $X$ be a fuzzy ordered set in which each nonempty fuzzy chain $C$ has a supremum and $X$ contains a least element $u$. If $F: X \rightarrow[0,1]^{X} \backslash\{\phi\}$ is a fuzzy multifunction which satisfies (I)-(III) as follows:

(I). Let there be a fuzzy order preserving fuzzy function $g: C \longrightarrow X$ such that $\{g(x)\} \subseteq F(x)$ for all $x \in C$. Then there exists $y_{0} \in F(\sup C)$ such that $\mu\left(g(x), y_{0}\right) \geq \mu\left(y_{0}, g(x)\right)$ for all $x \in C$.

(II). Let $\mu\left(x_{1}, x_{2}\right) \geq \mu\left(x_{2}, x_{1}\right)$ and let $y_{1} \in F\left(x_{1}\right), y_{2} \in F\left(x_{2}\right)$ with $\mu\left(y_{1}, y_{2}\right) \geq \mu\left(y_{2}, y_{1}\right)$. If $\mu\left(x_{1}, x\right) \geq$ $\mu\left(x, x_{1}\right)$ and $\mu\left(x, x_{2}\right) \geq \mu\left(x_{2}, x\right)$ then

$$
F(x) \cap\left\{z: \mu\left(y_{1}, z\right) \geq \mu\left(z, y_{1}\right) \text { and } \mu\left(z, y_{2}\right) \geq \mu\left(y_{2}, z\right)\right\} \neq \phi .
$$

(III). Let $D=\left\{z: \mu\left(x_{1}, z\right) \geq \mu\left(z, x_{1}\right)\right.$ and $\left.\mu\left(z, x_{2}\right) \geq \mu\left(x_{2}, z\right)\right\}$ for $\mu\left(x_{1}, x_{2}\right) \geq \mu\left(x_{2}, x_{1}\right)$. If $F(x) \cap D \neq$ $\phi$ then $\sup (D \cap F(x)) \in D \cap F(x)$.

Then there exists a fuzzy order preserving selector $f$ for $F$ on $X$.

Proof. Let $P$ be the collection of fuzzy subsets $Y$ of $X$ with properties:

(1). $u \in Y$.

(2). If $x \in Y$ and $\mu(z, x) \geq \mu(x, z)$ then $z \in Y$, and

(3). There is an order preserving fuzzy function $g: Y \longrightarrow X$ such that $g(x) \in F(x)$ for all $x \in Y$.

Let $(P, g)=\{(Y, g): \quad Y \in P$ and $g$ is a fixed fuzzy function from (3) $\}$. Define a fuzzy order on $(P, g)$ as follows: $\left(Y_{1}, g_{1}\right)<\left(Y_{2}, g_{2}\right)$ if and only if $Y_{1} \subseteq Y_{2}$ and $g_{1}=\left.g_{2}\right|_{Y_{1}}$. Then by fuzzy Zorn's lemma there is a maximal element $\left(X_{0}, f_{0}\right)$ of $(P, g)$. If $X=X_{0}$, we are done. Otherwise, suppose $x \in X \backslash X_{0}$, and let $C$ be a maximal fuzzy chain containing $u$ and $x$. Then $C \cap X_{0}=C_{1}$ is a fuzzy chain in $X_{0}$. Let $x_{0}=\sup C_{1}$. Condition (I) and maximality of $X_{0}$ imply that $x_{0} \in X_{0}$. Now pick a $y^{\prime} \in F(x)$ such that $\mu\left(f\left(x_{0}\right), y^{\prime}\right) \geq \mu\left(y^{\prime}, f\left(x_{0}\right)\right)$. Let $Y=X_{0} \cup\left\{z: \mu\left(x_{0}, z\right) \geq \mu\left(z, x_{0}\right)\right.$ and $\left.\mu(z, x) \geq \mu(x, z)\right\}$. Define $f: Y \longrightarrow Y$ as follows:

If $z \in X_{0}$, then $f(z)=f_{0}(z)$ and if $z \in\left\{z: \mu\left(x_{0}, z\right) \geq \mu\left(z, x_{0}\right)\right.$ and $\left.\mu(z, x) \geq \mu(x, z)\right\}$ then

$$
f(z)=\sup \left(F(z) \cap\left\{y: \mu\left(f\left(x_{0}\right), y\right) \geq \mu\left(y, f\left(x_{0}\right)\right) \text { and } \mu\left(y, y^{\prime}\right) \geq \mu\left(y^{\prime}, y\right)\right\}\right) .
$$

Set $f(x)=y^{\prime}$. Conditions (II) and (III) show that $f$ is well defined order preserving fuzzy function. This contradicts to the maximality of $X_{0}$. Hence $X=X_{0}$.

Theorem 4.3. Let $X$ be a fuzzy ordered set in which each nonempty fuzzy chain $C$ has a supremum. Let $F: X \longrightarrow[0,1]^{X} \backslash\{\phi\}$ be a fuzzy order preserving fuzzy multifunction such that, given a chain $C$ in $X$ and a single valued order preserving fuzzy function $g: C \longrightarrow X$ satisfying $\{g(x)\} \subseteq F(x)$ for all $x \in C$, there exists a $y_{0} \in F(\sup C)$ such that $\mu\left(g(x), y_{0}\right) \geq \mu\left(y_{0}, g(x)\right)$ for all $x \in C$. If there is a point $p \in X$ and $y \in F(p)$ such that $\mu(p, y) \geq \mu(y, p)$, then $F$ has a fixed point.

Proof. Let $p \in X$ and let $y \in F(p)$ with $\mu(p, y) \geq \mu(y, p)$. Define a collection $P$ of fuzzy subsets $Y$ of $X$ by:

(i). $p \in Y$.

(ii). If $\mu(p, z) \geq \mu(z, p), \mu(z, x) \geq \mu(x, z)$ and $x \in Y$ then $z \in Y$; and

(iii). If $x \in Y$, then there is a $z \in F(x)$ such that $\mu(x, z) \geq \mu(z, x)$.

Fuzzy order $P$ by inclusion. Since $\{p\} \in P$. Therefore by fuzzy Zorn's lemma there is a maximal element $X_{0} \in P$. Let $C$ be a maximal chain in $X_{0}$ (existence of $C$ is implied by fuzzy Zorn's lemma and let $x_{0}=\sup C$.

Element $x_{0} \in X_{0}$. Indeed; let there be a fuzzy subset $C_{0} \subseteq C$ such that :

(iv). $x_{0}=\sup C_{0}$, and

(v). There is an order preserving fuzzy function $g: C_{0} \longrightarrow X$ such that $\{g(x)\} \subseteq F(x)$ and $\mu(x, g(x)) \geq$ $\mu(g(x), x)$ for each $x \in C_{0}$.

Let $Q$ be the collection of fuzzy subsets of $C$ which satisfies (v). If $C_{1}, C_{2} \in Q$, then fuzzy order the pairs $\left(C_{1}, g_{1}\right),\left(C_{2}, g_{2}\right)$, where $g_{1}$ and $g_{2}$ are fixed fuzzy functions from condition (v), by $\left(C_{1}, g_{1}\right)<\left(C_{2}, g_{2}\right)$, if and only if $C_{1} \subseteq C_{2}$ and $g_{1}=\left.g_{2}\right|_{C_{1}}$. By fuzzy Zorn's lemma there is a maximal set $C_{0}$ with function $g_{0}$ in 
$Q$. Let $x^{\prime}=\sup C_{0}$. If $x^{\prime} \neq x_{0}$ then there is an $x \in C$ such that $\mu\left(x^{\prime}, x\right) \geq \mu\left(x, x^{\prime}\right)$. By hypothesis, we can extend $g_{0}$ to the set $C_{0} \cup\{x\}$ which contradicts the maximality of $C_{0}$. Thus $\sup C_{0}=x_{0}$. By hypothesis there is a $y \in F\left(x_{0}\right)$ such that $\mu(g(x), y) \geq \mu(y, g(x))$ for all $x \in C_{0}$. But $\mu(x, g(x)) \geq \mu(g(x), x)$ for $x \in C_{0}$ and so $y$ is an upper bound for $C_{0}$. Thus $\mu\left(x_{0}, y\right) \geq \mu\left(y, x_{0}\right)$. Hence, $x_{0} \in X_{0}$.

Since $x_{0} \in X_{0}$, there exists a $y_{0} \in F\left(x_{0}\right)$ such that $\mu\left(x_{0}, y_{0}\right) \geq \mu\left(y_{0}, x_{0}\right)$. If $x_{0}=y_{0}$, we are done. Otherwise suppose $\mu\left(x_{0}, y_{0}\right)>\mu\left(y_{0}, x_{0}\right)$. Then put $X_{1}=X_{0} \cup Z$, where $Z=\left\{z: \mu\left(x_{0}, z\right) \geq \mu\left(z, x_{0}\right)\right.$ and $\left.\mu\left(z, y_{0}\right) \geq \mu\left(y_{0}, z\right)\right\}$.

Since $F$ is fuzzy order preserving, therefore for each $z \in Z$, there is a $w \in F(z)$ such that $\mu\left(y_{0}, w\right) \geq$ $\mu\left(w, y_{0}\right)$. But then, $\mu(z, w) \geq \mu(w, z)$. Thus (i), (ii) and (iii) are satisfied by $X_{1}$, which contradicts the maximality of $X_{0}$. Hence, $x_{0}=y_{0}$ and thus $x_{0} \in F\left(x_{0}\right)$.

Remark 4.4. Theorem 4.3. generalizes/extends several known results including among them are Knaster [67], Tarski [86], Abian and Brown ([1], Theorem 2) and Beg ([5], Theorem 2.4). Also Theorem 4.1 is a fuzzy analogue of Smithson ([84, Theorem 1.1).

Remark 4.5. Results given in this section are due to Beg [10].

\section{FURTHER EXTENSION OF FUZZY ZORN'S LEMMA}

In economics, decision analysis, optimization and game theory, it is important to know under what conditions a relation has a maximal element on a nonempty set. Many results are given in the literature to prove the existence of maximal elements for a relation. Among these results, there are basically two streams: One stream assumes a convex cone preferences and focuses on a weakening the topological conditions (see Fan [47], Bergstrom [28] and Zhou and Tian [93]). The other stream assumes a certain nontransitive preference on a compact set with some other topological and/or convexity condition (see Corley [37] [38]). The Zorn's lemma [95] is a very powerful mathematical tool to avoid compactness assumption (see [2]). The main aim of this section is to further weaken the fuzzy transitivity condition in fuzzy Zorn's lemma without invoking any topological assumptions. A necessary and sufficient condition has been established to completely characterize the existence of maximal elements for general irreflexive nontransitive fuzzy relations.

Definition 5.1. Let $X$ be a set with a fuzzy relation $R$. A fuzzy subset $B$ of $X$ is said to be pointwisely dominated in $X$ if for each $x$ in $B$ there is some $y \in X$ such that $y \neq x$ and $r(x, y) \geq r(y, x)$. The fuzzy subset $B$ is called strictly dominated in $X$ if there is some $y \in X \backslash B$ such that $r(x, y)>r(y, x)=0$ for all $x \in B$. A pointwisely dominant R-fuzzy chain $C$ in $X$ is said to have the dominant property on $\mathrm{X}$, if it is strictly dominated in $X$. When every pointwisely dominated R-fuzzy chain $C \subset X$ is strictly dominated in $X$, we say that the fuzzy relation $R$ has fuzzy chain dominant property on $X$.

It is clear from the definition that, for a fuzzy relation $R$ on $X$ if there exists an element $x$ in $X$ such that $r\left(x, x_{*}\right)>r\left(x_{*}, x\right)=0$ for all $x$ in $X$ then $R$ has fuzzy chain dominant property on $X$. In a R-fuzzy chain $C$, the least and greatest elements are unique. A R-fuzzy chain $C_{1}$ in $(X, R)$ is said to be maximal chain if any R-fuzzy chain $C_{2}$ in $X$ with $C_{1} \subset C_{2}$ implies $C_{1}=C_{2}$.

Theorem 5.2. Let $R$ be a fuzzy relation on a nonempty set $X$ and let $x$ be an element in $X$. Then in $X$, there exists a maximal $R$-fuzzy chain above $x$.

Proof. Let $\mathcal{Z}$ be the set of all fuzzy chains in $X$ above $x$. Since $X$ is nonempty, $\mathcal{Z} \neq \phi$. For any two $C_{1}, C_{2} \in \mathcal{Z}$, if $C_{2} \subset C_{1}$ then we define a (partial) fuzzy order relation $Q$ on $\mathcal{Z}$ by $q\left(C_{2}, C_{1}\right)=\sup \left\{C_{1}(x)-\right.$ $\left.C_{2}(x): x \in X\right\}$. Then any chain in $\mathcal{Z}$ has an upper bound. Indeed, let $N$ be a chain in $\mathcal{Z}$; let $\mathcal{B}$ denote the set of all finite fuzzy subsets of $N$ ordered by $Q$. For each $B \in \mathcal{B}$, define: $\mathcal{C}_{B}=\cup\{c: c \in B\}$ and $\overline{\mathcal{C}}=\cup\left\{\mathcal{C}_{B}: B \in \mathcal{B}\right\}$. Now $\overline{\mathcal{C}}$ is an element of $\mathcal{Z}$ and $q(\mathcal{C}, \overline{\mathcal{C}})>q(\overline{\mathcal{C}}, C)$, for all $\mathcal{C} \in N$, i.e., $\overline{\mathcal{C}}$ is an upper bound of the chain $N$ in $\mathcal{Z}$. Applying theorem 2.7, we get a maximal element, say $\mathcal{C}^{*}$ in $\mathcal{Z}$. Hence $\mathcal{C}^{*}$ is the maximal R-fuzzy chain in $X$ containing $x$.

Theorem 5.3. Let $R$ be a fuzzy relation on a nonempty set $X$ having the chain dominant property then there exists a maximal element $x_{*}$ in $X$.

Proof. Since $X$ is nonempty, theorem 5.2 implies that there exists a maximal R-fuzzy chain $C^{*}$ (say) in $X$. If for each $z$ in $C^{*}$, there exists $x \in X$ such that $r(z, x) \geq r(x, z)$ Then $C^{*}$ is pointwisely dominated. By 
the fuzzy chain dominant property, there exists $\bar{x} \in X \backslash C^{*}$ such that $r(z, \bar{x})>r(\bar{x}, z)=0$ for all $z$ in $C^{*}$. In this case, $C^{*} \cup\{\bar{x}\}$ is again a R-fuzzy chain. Thus we may enlarge the R-fuzzy chain $C^{*}$ by adding $\bar{x}$ to $C$, and this will violate the maximality of the R-fuzzy chain $C^{*}$. Hence there exists an element $x_{*} \in C^{*}$ such that $r\left(x_{*}, x\right)=0$ for all $x$ in $X$ with $x \neq x_{*}$.

Next we prove that theorem 5.3 is equivalent to the fuzzy Zorn's lemma.

Theorem 5.4. Theorem 5.3 is equivalent to fuzzy Zorn's lemma.

Proof. To show the equivalence, we only need to prove that theorem 5.3 implies fuzzy Zorn's lemma.

Let $R^{*}$ be a fuzzy order relation on a nonempty set $X$. We define $p(y, x)>p(x, y)$ if $r^{*}(y, x) \geq r^{*}(x, y)$ and $x \neq y$. Then fuzzy relation $P$ is f-antisymmetric fuzzy relation. Indeed the fact that $p(y, x)>p(x, y)$ and $p(x, y)>p(y, x)$ imply $x \neq y, r^{*}(y, x) \geq r^{*}(x, y)$ and $r^{*}(y, x) \geq r^{*}(x, y)$. Therefore $x=y$ leads to a contradiction. The fuzzy relation $P$ is also f-transitive. For $p(y, x)>p(x, y)$ and $p(z, y)>p(y, z)$ imply $r^{*}(y, x) \geq r^{*}(x, y), r^{*}(z, y) \geq r^{*}(y, z)$. Thus $r^{*}(z, x) \geq r^{*}(x, z)$. Since $x=z$ is impossible, we have $p(z, x)>p(x, z)$.

Define the completion of $P$ by $P^{*}$, i.e., $p^{*}(y, x) \geq p^{*}(x, y)$ if $p(x, y) \ngtr p(y, x)$.

Next, we claim that, if each $R^{*}$-fuzzy chain in $X$ has an upper bound, then $P$ has the fuzzy chain dominant property on $X$. Let $C$ be a pointwise dominated $P$-fuzzy chain in $X$. Since $P$ implies $R^{*}$, it is clear that this $P$-fuzzy chain is also a $R$-fuzzy chain. By our assumption this chain has an upper bound $x_{0}$ (say) in $X$. Let $x_{1}, x_{2}$ be two elements from the chain with $p\left(x_{1}, x_{2}\right)>p\left(x_{2}, x_{1}\right)$; then $x_{0}=x_{1}$ will lead to $p\left(x_{0}, x_{2}\right)>p\left(x_{2}, x_{0}\right)$, a contradiction to the fact that $x_{0}$ is an upper bound of $C$. Therefore $p\left(x, x_{0}\right)>p\left(x_{0}, x\right)$ for all $x$ in $C$, except possibly $x_{0}=x_{2}$ for some element $x_{2}$, in the chain with $p\left(x, x_{2}\right)>p\left(x_{2}, x\right)$ for all $x$ in $C \backslash\left\{x_{2}\right\}$. If such $x_{2}$ does not exist in the chain, then $x_{0}$ is a dominator of the chain. If such $x_{2}$ does exist in the chain, then there are two possibilities: either $x_{0}=x_{2}$ and $p^{*}\left(x, x_{2}\right) \geq p^{*}\left(x_{2}, x\right)$ for all $x$ in $X$, or there exists $y_{0}$ in $X$ such that $p^{*}\left(x_{0}, y_{0}\right) \geq p^{*}\left(y_{0}, x_{0}\right)$ and $x_{0}=x_{2}$. The first case is impossible by our assumption, so we exclude it. The second case implies that $y_{0}$ is a dominator of the chain under the transitivity of $P$. So $P$ has the chain dominant property. Now theorem 5.3 guarantees the existence of a maximal element $x_{*}$ of $P$ on $X$ such that $p^{*}\left(x, x_{*}\right) \geq p^{*}\left(x_{*}, x\right)$ for all $x$ in $X$. Let $x \in X$ with $p^{*}\left(x_{*}, x\right) \geq p^{*}\left(x_{*}, x\right)$. Since $p\left(x_{*}, x\right)>p\left(x_{*}, x\right)$ will violate $p^{*}\left(x, x_{*}\right) \geq p^{*}\left(x_{*}, x\right)$ for all $x$ in $X$. Thus we have $x=x_{*}$. Hence $x_{*}$ is a maximal element of fuzzy order relation $R^{*}$ on $X$.

Remark 5.5. Theorem 5.3 is an extension of the fuzzy Zorn's lemma to general nontransitive fuzzy relations without invoking any topological assumptions or linear structure. We expect that theorem 5.3 can be used for optimization, when the fuzzy relation is nontransitive for which the original fuzzy Zorn's lemma fails to fit.

Remark 5.6. Results given in this section are due to Beg [11].

\section{FUZZY ORDERING AND COMPLETENESS OF FUZZY METRIC SPACES}

Ekeland's variational principle [45] [46] and its equivalence theorem, and Caristi's fixed point theorem [33 [34 [66] are very useful in nonlinear analysis, control theory, global analysis and geometric theory of metric spaces and Banach spaces. Central to the proof of all these is an argument based on completeness. Several other authors have also exploited these ideas in other areas (see [50, [92]] and references therein). Zadeh [91] and Negoita and Ralescu [74] have introduced the notion of fuzzy order and similarity in their papers, which was subsequently further developed and refined by Venugopalan [87, Billot [29] and Beg et al [25] [26] 27]. Recently Kaleva and Seikkala [62] introduced the concept of a fuzzy metric space which generalize the notion of metric space by setting the distance between two points to be a non-negative fuzzy number, and investigated their properties. Afterwards fuzzy analogue of fixed point theorems and Ekeland's variational principle in fuzzy metric spaces were studied by Kaleva [61, Hadzic [51], Fang [48], He [53], Jung, Cho, Kang and Chang [60], Beg [5] and Vijayaraju and Marudai [88]. Our aim in this section is to elucidate the connection between fixed point theorem and the basic completeness technique in fuzzy metric spaces. We show that fuzzy Ekeland theorem is a consequence of Beg ([9], Theorem 7) and it further characterizes 
complete fuzzy metric spaces. We also obtained a characterization in terms of existence of fixed points in fuzzy metric spaces.

Beg ([9], Theorem 7) can be reformulated as follows:

Theorem 6.1. Suppose that $X$ is a fuzzy ordered set such that every decreasing chain in $X$ has a greatest lower bound in $X$. If $f: X \rightarrow X$ is a map such that for all $x$ in $X, \mu(f(x), x) \geq \mu(x, f(x))$, then there is $a v$ in $X$ with $f(v)=v$.

The concept of fuzzy metric space was introduced by Kaleva and Seikkala [62]. For the sake of completeness we state some definitions and results (6.2-6.6) from [62.

We denote $\mathbb{R}=(-\infty, \infty), \mathbb{R}_{+}=[0, \infty)$, the set of all upper semicontinuous normal convex fuzzy numbers by $E$ and the set of all non-negative fuzzy numbers in $E$ by $G$.

The additive and multiplicative identities of fuzzy numbers are denoted by $\overline{0}$ and $\overline{1}$, respectively.

The $\alpha$-level set $[x]_{\alpha}$ of a fuzzy number $x \in E$ is a closed interval $[a, b]$, where the values $a=-\infty$ and $b=\infty$ are admissible.

Definition 6.2. An ordering $\leq$ in $E$ is defined by $x \leq y$ if and only if $a_{1}^{\alpha} \leq a_{2}^{\alpha}$ and $b_{1}^{\alpha} \leq b_{2}^{\alpha}$ for all $\alpha \in(0,1]$, where $x, y \in E,[x]_{\alpha}=\left[a_{1}^{\alpha}, b_{1}^{\alpha}\right],[y]_{\alpha}=\left[a_{2}^{\alpha}, b_{2}^{\alpha}\right]$.

Definition 6.3. A sequence $\left\{x_{n}\right\}$ in $E$ is called $\alpha$-level convergent to $x \in E$, if

$$
\lim _{n \rightarrow \infty} a_{n}^{\alpha}=a^{\alpha} \text { and } \lim _{n \rightarrow \infty} b_{n}^{\alpha}=b^{\alpha} \quad \text { for all } \alpha \in(0,1],
$$

where $\left[x_{n}\right]_{\alpha}=\left[a_{n}^{\alpha}, b_{n}^{\alpha}\right]$ and $[x]_{\alpha}=\left[a^{\alpha}, b^{\alpha}\right]$.

In the following, the set $E$ will be endowed with the above ordering and the $\alpha$-level convergence.

Definition 6.4. Let $X$ be a non-empty set, $d$ a mapping from $X \times X$ into $G$ and let the mappings $L, R$ : $[0,1] \times[0,1] \rightarrow[0,1]$ be symmetric, non-decreasing in both arguments and satisfy $L(0,0)=0, R(1,1)=1$. Denote

$$
[d(x, y)]_{\alpha}=\left[\lambda_{\alpha}(x, y), \rho_{\alpha}(x, y)\right]
$$

for $x, y \in X, \alpha \in(0,1]$.

The quadruple $(X, d, L, R)$ is called a fuzzy metric space and $d$ a fuzzy metric, if

(i) $d(x, y)=\overline{0}$ if and only if $x=y$,

(ii) $d(x, y)=d(y, x)$ for all $x, y \in X$,

(iii) for all $x, y, z \in X$,

(1) $\quad d(x, y)(s+t) \geq L(d(x, z)(s), d(z, y)(t))$ whenever $s \leq \lambda_{1}(x, z), \quad t \leq \lambda_{1}(z, y)$ and $s+t \leq \lambda_{1}(x, y)$,

(2) $\quad d(x, y)(s+t) \leq R(d(x, z)(s), d(z, y)(t))$ whenever $s \geq \lambda_{1}(x, z), t \geq \lambda_{1}(z, y)$ and $s+t \geq \lambda_{1}(x, y)$.

The following 2-place functions are some frequently used choices for $L$ and $R$ :

$T_{1}(a, b)=\max (a+b-1,0), T_{2}(a, b)=a b, T_{3}(a, b)=\min (a, b)$,

$T_{4}(a, b)=\max (a, b), T_{5}(a, b)=a+b-a b$ and $T_{6}(a, b)=\min (a+b, 1)$.

Theorem 6.5. Let $(X, d, L, R)$ be a fuzzy metric space with $\lim _{a \rightarrow 0+} R(a, a)=0$. Then the family $\beta=\{(\beta(\varepsilon, \alpha): \varepsilon>0, \quad 1 \geq \alpha>0\}$ of sets

$\beta(\varepsilon, \alpha)=\left\{(x, y) \in X \times X: \rho_{\alpha}(x, y)<\varepsilon\right\}$ forms a basis for a Hausdorff uniformity on $X \times X$. Moreover, the sets

$N_{x}(\varepsilon, \alpha)=\left\{y \in X: \rho_{\alpha}(x, y)<\varepsilon\right\}$ form a basis for a Hausdorff topology on $X$ and this topology is metrizable.

We call the topology on $X$, determined by above basis, a fuzzy metric topology, and denote it $\mathcal{T}_{d}$.

Theorem 6.6. In fuzzy metric space $(X, d, L, R)$ with $R=\max$, the triangle inequality (iii) (2), in Definition 6.4, is equivalent to

$$
\begin{gathered}
\rho_{\alpha}(x, y) \leq \rho_{\alpha}(x, z)+\rho_{\alpha}(z, y), \\
x, y, z \in X, \alpha \in(0,1] .
\end{gathered}
$$


The convergence in a fuzzy metric space $(X, d, L, R)$ is defined by $\lim _{n \rightarrow \infty} x_{n}=x$ if and only if $\lim _{n \rightarrow \infty} d\left(x_{n}, x\right)=\overline{0}$. From the definition of convergence in $G$ and Theorem 6.5 , it follow that in the fuzzy metric space $(X, d, L, R)$ with $\lim _{a \rightarrow 0+} R(a, a)=0$, the limit is determined uniquely and all subsequences of a convergent sequence converge. A sequence $\left\{x_{n}\right\}$ in $X$ is called a Cauchy sequence if $\lim _{m, n \rightarrow \infty} d\left(x_{m}, x_{n}\right)=\overline{0}$. A fuzzy metric space $X$ is complete if every Cauchy sequence in $X$ converges. From the inequality (1), it follows that in the fuzzy metric space $(X, d, L, \max )$, every convergent sequence is also a Cauchy sequence.

Next, we prove Ekeland's variational principle in fuzzy metric spaces.

Theorem 6.7.[Ekeland's variational principle]. Let $(X, d, L$, max) be a complete fuzzy metric space such that $\lim _{t \rightarrow \infty} d(x, y)(t)=0$, for all $x, y \in X$. Let $F: X \rightarrow \mathbb{R}$ be a real-valued continuous function on $X$ such that $\inf F>-\infty$. Then, for each $\varepsilon>0$, there is a $v$ in $X$ such that for all $w \neq v$,

$$
F(w)+\varepsilon \rho_{\alpha}(w, v)>F(v),
$$

$\alpha \in(0,1]$.

Proof. Since $\lim _{t \rightarrow \infty} d(x, y)(t)=0$, it follows that $\rho_{\alpha}(x, y)<\infty$ for all $x, y \in X$ and $\alpha \in(0,1]$. Therefore by using the triangle inequality (1) from definition 6.4 , we can define a fuzzy order $\eta_{F, \varepsilon}$ on $X$ by

$$
\eta_{F, \varepsilon}(x, y) \geq \eta_{F, \varepsilon}(y, x) \text { if and only if } F(x)+\varepsilon \rho_{\alpha}(x, y) \leq F(y) \text {. }
$$

The fact that every decreasing chains have greatest lowers bounds follows from completeness and the fact that $F$ is continuous and bounded below. If $\left(x_{\delta}\right)$ decreases, then $F\left(x_{\delta}\right)$ non-increases and converges. Since, for $\delta<\beta$, we have

$$
\varepsilon \rho_{\alpha}\left(x_{\delta}, x_{\beta}\right) \leq F\left(x_{\delta}\right)-F\left(x_{\beta}\right),
$$

the net $\left(x_{\delta}\right)$ is Cauchy and converges to the greatest lower bound, $x$.

Define the map $f: X \rightarrow X$ by

$$
f(x)= \begin{cases}y, & \text { if } \eta_{F, \varepsilon}(y, x)>\eta_{F, \varepsilon}(x, y), \\ x, & \text { otherwise }\end{cases}
$$

Now Theorem 6.1 implies there must be a fixed point, $v$, for $f$. Hence if $w \neq v$ we cannot have $\eta_{F, \varepsilon}(w, v) \geq$ $\eta_{F, \varepsilon}(v, w)$ and so $F(w)+\varepsilon \rho_{\alpha}(w, v)>F(v)$.

Corollary 6.8. Let $(X, d, L, \max )$ be a complete fuzzy metric space such that $\lim _{t \rightarrow \infty} d(x, y)(t)=0$, for all $x, y \in X$. Let $F: X \rightarrow \mathbb{R}$ be a real-valued continuous function on $X$ such that $\inf F>-\infty$ and $u \in X$ is arbitrary. Then for each $\varepsilon>0$ there is a $v \in X$ such that

(i) $F(v)+\varepsilon \rho_{\alpha}(v, u) \leq F(u)$, and

(ii) for all $w \neq v, \quad F(w)+\rho_{\alpha}(w, v)>F(v)$,

where $\alpha \in(0,1]$.

Proof. The complete fuzzy metric subspace $\left\{x: \eta_{F, \varepsilon}(x, u) \geq \eta_{F, \varepsilon}(u, x)\right\}$, satisfies all the necessary conditions of Theorem 6.7. Therefore there exists a $v \in X$ satisfying (i) and (ii) for all $w \neq v$ such that $\eta_{F, \varepsilon}(w, u) \geq$ $\eta_{F, \varepsilon}(u, w)$. However if the inequality in (ii) is not satisfied by some $w \in X$, then $\eta_{F, \varepsilon}(w, v) \geq \eta_{F, \varepsilon}(v, w)$ and so $\eta_{F, \varepsilon}(w, u) \geq \eta_{F, \varepsilon}(u, w)$. Hence $w=v$.

Remark 6.9. (a). Using a more careful construction, as in He [53], we can show that Corollary 6.8. holds for lower semi continuous functions $\phi: X \rightarrow \mathbb{R}$.

(b). If $u \in X$ and $F(u) \leq \inf F+\delta$ for some $\delta>0$, then Corollary 6.8, implies that there exists a $v \in X$ satisfying (i) and (ii) and $\rho_{\alpha}(u, v) \leq \frac{\delta}{\varepsilon}$. 
Now we make use of our results to characterize complete fuzzy metric spaces in terms of existence of fixed points.

Theorem 6.10. Suppose $(X, d, L$, max $)$ is a fuzzy metric space such that

$$
\lim _{t \rightarrow \infty} d(x, y)(t)=0, \text { for all } x, y \in X .
$$

The fuzzy metric space $X$ is complete if and only if for every continuous function $F: X \rightarrow \mathbb{R}$ with inf $F \geq 0$ and every $\varepsilon>0$ and each $u \in X$ such that $F(u) \leq$ inf $F+\varepsilon$, there is a $v \in X$ such that

(i) $F(v) \leq F(u)$, and

(ii) $F(w)+\varepsilon \rho_{\alpha}(w, u) \geq F(v)$

for all $w \in X$ and $\alpha \in(0,1]$.

Proof. Suppose that fuzzy metric space $X$ is complete then Corollary 6.8 implies the existence of a $v \in X$ with the desired properties.

Conversely, suppose that $\left(x_{n}\right)$ is a Cauchy sequence in fuzzy metric space $X$ and consider the continuous function $F: X \rightarrow \mathbb{R}$ given by $F(y)=\lim _{n} \rho_{\alpha}\left(x_{n}, y\right)$. Since the sequence $\left(x_{n}\right)$ is Cauchy, inf $F=0$. Let $0<\varepsilon<1$ and $u \in X$ such that $F(u) \leq \varepsilon$.

By hypothesis, there is a $v \in X$ satisfying (i) and (ii). Since $F(v) \leq \varepsilon$. Thus for any $\eta>0$ there is an $x_{n}$ (for $n$ sufficiently large) such that $\rho_{\alpha}\left(x_{n}, v\right)<\varepsilon+\eta$ and $F\left(x_{n}\right)<\eta$. Condition (ii) of hypothesis further implies that

$$
\eta+\varepsilon(\varepsilon+\eta)>F\left(x_{n}\right)+\varepsilon \rho_{\alpha}\left(x_{n}, v\right) \geq F(v) .
$$

Since $\eta>0$ was arbitrary, therefore $\varepsilon^{2} \geq F(v)$. By repeating the same argument, we obtain $\varepsilon^{n} \geq F(v)$, for $n \geq 2$. It further implies that $F(v)=0$. i.e. $\lim _{n} x_{n}=v$. Hence $X$ is a complete fuzzy metric space.

Next we prove a characterization of completeness of a fuzzy metric space in terms of existence of fixed points and obtain an analogue of the results of Caristi and Kirk [34] and Kirk [66] in fuzzy metric spaces.

Theorem 6.11. Suppose $(X, d, L, \max )$ is a fuzzy metric space such that

$$
\lim _{t \rightarrow \infty} d(x, y)(t)=0 \text { for all } x, y \in X .
$$

The fuzzy metric space $X$ is complete if and only if for each continuous function $F: X \rightarrow \mathbb{R}$ with inf $F>-\infty$ and each $\varepsilon>0$ every map $f: X \rightarrow X$ satisfying $\eta_{F, \varepsilon}(f(x), x) \geq \eta_{F, \varepsilon}(x, f(x))$, has a fixed point $v$ and $\eta_{F, \varepsilon}(v, u) \geq \eta_{F, \varepsilon}(u, v)$ for any $u$ with $F(u) \leq$ inf $F+\varepsilon$.

Proof. Let $X$ be a complete fuzzy metric space. Then the $v$, whose existence is guaranteed by Corollary 6.8 is a fixed point of $f$. Otherwise if $f(v) \neq v$, then by condition (ii) of Corollary 6.8, we have $F(f(v))+$ $\varepsilon \rho_{\alpha}(v, f(v))>F(v)$. Hence a contradiction to $\eta_{F, \varepsilon}(f(v), v) \geq \eta_{F, \varepsilon}(v, f(v))$.

Conversely, assume that $X$ has fixed point property and $F, \varepsilon, u$ are given. Define $f: X \rightarrow X$ by

$$
f(x)= \begin{cases}y & \text { if } \eta_{F, \varepsilon}(y, x)>\eta_{F, \varepsilon}(x, y) \\ x & \text { otherwise }\end{cases}
$$

A fixed point $v$ of $f, \eta_{F, \varepsilon}(v, u) \geq \eta_{F, \varepsilon}(u, v)$ satisfies conditions (i) and (ii) of Theorem 6.10. and it further implies that the fuzzy metric space $(X, d, L, \max )$ is complete.

Remark 6.12. Results presented in this section are due to Beg [12].

\section{EXTENSION OF FUZZY POSITIVE LINEAR OPERATOR}

In his seminal paper Zadeh [90] studied the geometric form of the Hahn-Banach theorem in the case of finite dimension. Fuzzy set theory has developed rapidly in last three decades but surprisingly (to the best of our knowledge) no attempt has been made to obtain fuzzy analogue of the famous Hahn - Banach theorem 
and its consequences. Zadeh [91] gave the notion of fuzzy order which was further studied by Venugopalan [87. Recently using this notion of fuzzy order, Beg et al [4] [25] [26] [27] have defined fuzzy Riesz spaces and studied their properties. The aim of this section is to study the extension of fuzzy positive linear operators defined on a majorizing vector subspace of a fuzzy Riesz space with values in a complete fuzzy Riesz space. The fuzzy analogue of the most general form of the famous Hahn-Banach theorem is also given.

We modify few definitions for use in rest of this paper. Note difference between definition 7.1 and definition 2.2 .

Definition 7.1. Let $A$ be a crisp subset of a fuzzy ordered set $X$, then the upper bound $U(A)$ of $A$ is the fuzzy set on $\mathrm{X}$ defined as follows:

$$
U(A)(y)= \begin{cases}o & \text { if } \mu(x, y) \leq \frac{1}{2} \text { for some } \quad x \in A \\ \bigwedge_{x \in A} \mu(x, y) & \text { otherwise. }\end{cases}
$$

The lower bound $L(A)$ of $\mathrm{A}$ is the fuzzy set on $\mathrm{X}$ defined by:

$$
L(A)(y)= \begin{cases}o & \text { if } \mu(y, x) \leq \frac{1}{2} \text { for some } x \in A \\ \bigwedge_{x \in A} \mu(y, x) & \text { otherwise. }\end{cases}
$$

An element $z$ is called supremum of $A$ if (i) $z \in U(A)$ and (ii) if $y \in U(A)$ then $y \in U(z)$. The infimum is defined similarly.

Definition 7.2. A subset $D$ of a fuzzy ordered set $X$ is said to be directed if for every finite subset $F$ of $D$ ,$D \cap U(F) \neq \phi$ and $D \cap L(F) \neq \phi$.

Definition 7.3. A real linear space $X$ is said to be a fuzzy ordered linear space if $\mathrm{X}$ is a fuzzy ordered set and

(i) if $x_{1}, x_{2} \in X \quad$ such that $\mu\left(x_{1}, x_{2}\right)>\frac{1}{2}$ then $\mu\left(x_{1}, x_{2}\right) \leq \mu\left(x_{1}+x, x_{2}+x\right)$ for all $x \in X$;

(ii) if $x_{1}, x_{2} \in X \quad$ such that $\mu\left(x_{1}, x_{2}\right)>\frac{1}{2}$ then $\mu\left(x_{1}, x_{2}\right) \leq \mu\left(\alpha x_{1}, \alpha x_{2}\right)$ for all $0<\alpha \in \mathbb{R}$.

In a fuzzy ordered linear space $X$, an element $x$ is said to be fuzzy positive if $\mu(0, x)>\frac{1}{2}$, it is called fuzzy negative if $\mu(x, 0)>\frac{1}{2}$ and $X_{+}$denotes the set of all fuzzy positive elements of $X$. If $\mathrm{x}$ is an arbitrary element of $X$ then the positive part of $\mathrm{x}$ is the element $x_{+}=x \vee 0$, the negative part $x_{-}=(-x) \vee_{0}$, the absolute value of $\mathrm{x}$ is the element $|x|=x \vee(-x)$.

Definition 7.4.(i). A fuzzy ordered linear space which is a directed set is called a directed fuzzy ordered linear space. (ii). A directed fuzzy ordered linear space $X$ is called a fuzzy Archimedean space, if for any non-negative element $x$, the set $\{\alpha x: 0<\alpha \in \mathbb{R}\}$ is not bounded from above.

Definition 7.5. A fuzzy ordered linear space $X$ is called a fuzzy Riesz space if all finite subsets of $X$ have supremum and infimum. The space $X$ is complete if every bounded subset of $X$ has supremum and infimum.

We now state without proof 7.6 to 7.9 , whose proofs can be derived easily (see 25] 26] [27]).

Proposition. 7.6. Let $\left\{x_{i}\right\}_{i \in J}$ and $\left\{y_{k}\right\}_{k \in K}$ be two systems of elements of a fuzzy ordered linear space. If $\bigvee_{i \in J} x_{i}$ and $\bigvee_{k \in K} y_{k}$ exist, then $\bigvee_{i \in J, k \in K}\left(x_{i}+y_{k}\right)$ exists too and $\bigvee_{i \in J, k \in K}\left(x_{i}+y_{k}\right)=\bigvee_{i \in J} x_{i}+\bigvee_{k \in K} y_{k}$.

An analogous result holds for the greatest lower bounds.

Proposition 7.7. A directed fuzzy ordered linear space $X$ is fuzzy Archimedean space if and only if: for $x, y \in X$ such that $\mu\left(\alpha_{n} x, y\right)>\frac{1}{2}$ and $\alpha_{n} \longrightarrow \alpha$ one should infer that $\mu(\alpha x, y)>\frac{1}{2}$.

Theorem 7.8. In a fuzzy Riesz space $X, \mu(|x+y|,|x|+|y|)>\frac{1}{2}$. 
Lemma 7.9. For any positive elements $x, x_{1}, x_{2}$ in a fuzzy Riesz space $X$,

$$
\mu\left(x \wedge\left(x_{1}+x_{2}\right), x \wedge x_{1}+x \wedge x_{2}\right)>\frac{1}{2} .
$$

Definition 7.10. An operator $U$, which maps a fuzzy ordered linear space $X$ into a fuzzy ordered linear space $Y$, is said to be fuzzy positive if and only if $\mu(0, x)>\frac{1}{2}$ implies $\eta(0, U(x)) \geq \mu(0, x)$. ( where $\eta$ is fuzzy order on $Y$ ).

Remark 7.11. (i). If $U: X \longrightarrow Y$ is an additive fuzzy positive operator then $\mu\left(x_{1}, x_{2}\right)>\frac{1}{2}$ implies $\eta\left(U\left(x_{1}\right), U\left(x_{2}\right)\right) \geq \mu\left(x_{1}, x_{2}\right)$.

(ii). Let $X$ and $Y$ be two fuzzy Riesz spaces and $U: X \longrightarrow Y$ an additive fuzzy positive operator, then Theorem 7.8 implies

$$
\eta\left(\left|U\left(x_{+}\right)-U\left(x_{-}\right)\right|, \quad\left|U\left(x_{+}\right)\right|+\left|U\left(x_{-}\right)\right|\right)>\frac{1}{2} .
$$

Therefore for $x \in X$,

$$
\eta(|U(x)|, \quad U(|x|))>\frac{1}{2}
$$

Lemma 7.12. Let $X$ be a fuzzy Riesz space and $Y$ be a fuzzy Archimedean space. If $U: X \longrightarrow Y$ is an additive fuzzy positive operator then $U$ is homogeneous.

Proof. Let $\alpha$ be any real number, then there exists sequences $\left\{a_{n}\right\}_{n \in N}$ and $\left\{b_{n}\right\}_{n \in N}$ of rational numbers such that $a_{n} \uparrow \alpha$ and $b_{n} \downarrow \alpha$. Consider any nonzero positive element $x$ is $X$, then

$$
\mu\left(a_{n} x, \alpha x\right)>\frac{1}{2} \text { and } \mu\left(\alpha x, b_{n} x\right)>\frac{1}{2} .
$$

It implies that, $\eta\left(U\left(a_{n} x\right), U(\alpha x)\right)>\frac{1}{2}$ and $\eta\left(U(\alpha x), U\left(b_{n} x\right)\right)>\frac{1}{2}$. Therefore $\eta\left(a_{n} U(x), U(\alpha x)\right)>\frac{1}{2}$ and $\eta\left(U(\alpha x), b_{n} U(x)\right)>\frac{1}{2}$. Proposition 7.7 further implies that $\eta(\alpha U(x), U(\alpha x))>\frac{1}{2}$ and $\eta(U(\alpha x), \alpha U(x))>\frac{1}{2}$ . Hence $U(\alpha x)=\alpha U(x)$.

Lemma 7.13. Let $X$ be a fuzzy Riesz space and $Y$ be a vector space. If $U: X_{+} \longrightarrow Y$ satisfies

$$
U(x+y)=U(x)+U(y)
$$

for $x, y \in X_{+}$, then $U$ extends uniquely to an additive operator $V$ from $X$ into $Y$. Moreover,

$$
V(x)=U\left(x_{+}\right)-U\left(x_{-}\right)
$$

for each $x \in X$.

Proof. Equality (2) defines an addition operator on the fuzzy Riesz space $X$. Indeed, let $x, y \in X$, then there exists an element $a \geq 0$ of $X$ such that

$$
\begin{aligned}
(x+y)_{+} & +a=x_{+}+y_{+} \\
\text {and } \quad(x+y)_{-} & +a=x_{-}+y_{-} .
\end{aligned}
$$

Hence

$$
\begin{aligned}
& U\left((x+y)_{+}\right)+U(a)=U\left(x_{+}\right)+U\left(y_{+}\right) . \\
& U\left((x+y)_{-}\right)+U(a)=U\left(x_{-}\right)+U\left(y_{-}\right) .
\end{aligned}
$$

It further implies,

$$
V(x+y)=V(x)+V(y) .
$$


Obviously $V$ is a unique extension of $U$.

Let $f$ and $g$ be two linear functional on a fuzzy Riesz space $X$, then $f \leq g$ if and only if $g-f$ is fuzzy positive .

Theorem 7.14. Let $f$ be a fuzzy positive linear functional on a fuzzy Riesz space $X$, then for any $x \in X$,

$$
f\left(x_{+}\right)=\sup \left\{g(x): g \in X^{\star} \text { and } 0 \leq g \leq f\right\},
$$

where $X^{\star}$ is the algebraic dual of the linear space $X$.

Proof. Let $a \in X$ then

$$
\sup \left\{g(a): g \in X^{\star} \text { and } 0 \leq g \leq f\right\} \leq f(a) \leq f\left(a_{+}\right) .
$$

Define the functional $U$ on $X_{+}$by

$$
U(x)=\sup \left\{f\left(x \wedge \alpha a_{+}\right): 0 \leq \alpha \in \mathbb{R}\right\},
$$

then for $x_{1}, x_{2} \in X_{+}, U\left(x_{1}+x_{2}\right)=U\left(x_{1}\right)+U\left(x_{2}\right)$. Indeed, for any $\alpha \geq 0$, Lemma 7.9 implies that

$$
\mu\left(\left(x_{1}+x_{2}\right) \wedge \alpha a_{+},\left(x_{1} \wedge \alpha a_{+}\right)+\left(x_{2} \wedge \alpha a_{+}\right)\right)>\frac{1}{2} .
$$

Therefore

$$
\eta\left(f\left(\left(x_{1}+x_{2}\right) \wedge \alpha a_{+}\right), f\left(x_{1} \wedge \alpha a_{+}\right)+f\left(x_{2} \wedge \alpha a_{+}\right)\right)>\frac{1}{2},
$$

where $\eta$ is a fuzzy order relation on $\mathbb{R}$. It further implies that

$$
\eta\left(U\left(x_{1}+x_{2}\right), U\left(x_{1}\right)+U\left(x_{2}\right)\right)>\frac{1}{2} .
$$

On the other hand, Proposition 7.6 implies that for $\alpha_{1}, \alpha_{2} \geq 0$,

$$
\mu\left(\left(x_{1} \wedge \alpha_{1} a_{+}\right)+\left(x_{2} \wedge \alpha_{2} a_{+}\right),\left(x_{1}+x_{2}\right) \wedge\left(\alpha_{1}+\alpha_{2}\right) a_{+}\right)>\frac{1}{2} .
$$

It further implies that

$$
\eta\left(f\left(x_{1} \wedge \alpha_{1} a_{+}\right)+f\left(x_{2} \wedge \alpha_{2} a_{+}\right), f\left(\left(x_{1}+x_{2}\right) \wedge\left(\alpha_{1}+\alpha_{2}\right) a_{+}\right)\right)>\frac{1}{2} .
$$

Thus $\eta\left(U\left(x_{1}\right)+U\left(x_{2}\right), U\left(x_{1}+x_{2}\right)\right)>\frac{1}{2}$. Hence $U\left(x_{1}+x_{2}\right)=U\left(x_{1}\right)+U\left(x_{2}\right)$.

Lemma 7.13. implies that $U$ can be uniquely extended to an additive functional $V$ defined on $X$ which is also positive.

Since for $x \in X_{+}, V(x)=U(x) \leq f(x)$, Therefore $V \leq f$. It follows that $0 \leq V \leq f$ and $V \in X^{*}$. Since $V\left(a_{-}\right)=0$, it follows that

$$
V(a)=U\left(a_{+}\right)=f\left(a_{+}\right)<\sup \left\{g(a): g \in X^{*} \text { and } 0 \leq g \leq f\right\} .
$$

Hence

$$
f\left(a_{+}\right)=\sup \left\{g(a): g \in X^{*} \text { and } 0 \leq g \leq f\right\}
$$

Definition 7.15. A set $S$ of fuzzy positive linear functionals on a fuzzy Riesz space $X$ is called sufficient if for any nonzero $x \in X$ there exists an $f \in S$ such that $f(x) \neq 0$.

Theorem 7.16. Let $X$ be a fuzzy Riesz space with a sufficient set of fuzzy positive linear functionals. Let $a \in X$. If $\eta(0, f(a))>\frac{1}{2}$ for every fuzzy positive linear functional $f$ then $\mu(0, a)>\frac{1}{2}$. 
Proof. Let $f$ and $g$ be linear functionals on $X$ and $0 \leq g \leq f$ then $\eta(g(a), f(a))>\frac{1}{2}$. Theorem 7.14. implies that $f\left(a_{+}\right)=f(a)$, for every fuzzy positive linear functional $f$. Since on $X$ there exists a sufficient set of fuzzy positive linear functionals, therefore $a=a_{+}$. Hence $\mu(0, a)>\frac{1}{2}$.

Definition 7.17. A vector subspace $Z$ of a fuzzy Riesz space $X$ is said to be a majorizing vector subspace, if for any $x \in X$, there exist $x_{1}, x_{2} \in Z$ such that $\mu\left(x_{1}, x\right)>\frac{1}{2}$ and $\mu\left(x, x_{2}\right)>\frac{1}{2}$.

Theorem 7.18. Let $Z$ be a majorizing vector subspace of a fuzzy Riesz space $X$ and $Y$ a complete fuzzy Riesz space. If $U: Z \longrightarrow Y$ is a fuzzy positive linear operator then there exists $V: X \longrightarrow Y$ a fuzzy positive linear operator such that $V(x)=U(x)$ for $x \in Z$.

Proof. Let $v \in Z^{c}$ and $x_{1}, x_{2} \in Z$ be such that $\mu\left(x_{1}, v\right)>\frac{1}{2}$ and $\mu\left(v, x_{2}\right)>\frac{1}{2}$. Since $U$ is a fuzzy positive linear operator therefore

$$
\eta\left(U\left(x_{1}\right), U\left(x_{2}\right)\right)>\frac{1}{2} \text {. }
$$

Define

$$
\begin{gathered}
a=\sup \left\{U(x): x \in Z \text { and } \mu(x, v)>\frac{1}{2}\right\}, \\
b=\inf \left\{U(x): x \in Z \text { and } \mu(v, x)>\frac{1}{2}\right\} .
\end{gathered}
$$

and

$$
y_{1} \in[a, b] .
$$

Let $X_{1}=\operatorname{span}(Z \cup\{v\})$. Define $U_{1}: X_{1} \longrightarrow Y$ by $U_{1}(x+\alpha v)=U(x)+\alpha y_{1}$, where $x \in Z$ and $\alpha \in \mathbb{R}$.

The operator $U_{1}$ is a fuzzy positive linear operator. Indeed, let $\mu(0, x+\alpha v)>\frac{1}{2}$ where $\alpha \neq 0$. If $\alpha>0$, then $\mu\left(-\frac{1}{\alpha} x, v\right)>\frac{1}{2}$. It further implies $\eta\left(-\frac{1}{\alpha} U(x), y_{1}\right)>\frac{1}{2}$. Thus $\eta\left(0, U_{1}(x+\alpha v)\right)>\frac{1}{2}$. If $\alpha<0$, we can prove similarly.

Let $B$ be the set of all fuzzy positive linear extensions of $U$. Order $\mathbf{B}$ by putting $V_{1} \leq V_{2}$ if and only if $V_{2}$ is an extension of $V_{1}$. Then $\mathbf{B}$ satisfies conditions of Zorn's lemma. Therefore there exists a maximal element $V$ defined on the whole space $X$.

The next result is the fuzzy version of the most general form of what is known as the Hahn-Banach extension theorem.

Theorem 7.19. [Fuzzy Hahn-Banach Theorem] Let $X$ be a (real) vector space, $Y$ a complete fuzzy Archimedean Riesz space, and let $p: X \longrightarrow Y$ be a sublinear operator. If $Z$ is a subspace of $X$ and $U: Z \longrightarrow Y$ is a linear operator satisfying $\eta(S(x), p(x))>\frac{1}{2}$ for all $x \in Z$ then there exists $V: X \longrightarrow Y a$ linear operator such that $V(x)=U(x)$ for $x \in Z$ and $\eta(V(x), p(x))>\frac{1}{2}$ for $x \in X$.

Proof. Let $v \in Z^{c}$ and let $X_{1}=\operatorname{span}\{Z \cup\{v\}\}$. If $U_{1}: X_{1} \longrightarrow Y$ is an extension of $U$, then for $z \in Z$ and $\lambda \in \mathbb{R}$,

$$
U_{1}(z+\lambda v)=U(z)+\lambda y_{1}
$$

and

$$
\eta\left(U(z)+\lambda y_{1}, p(z+\lambda v)\right)>\frac{1}{2}
$$

where $y_{1}=U_{1}(v)$.

Indeed, for $\lambda>0,(3)$ is equivalent to

$$
\eta\left(U(z)+y_{1}, p(z+v)\right)>\frac{1}{2}
$$

for all $z \in Z$, while for $\lambda<0,(3)$ is equivalent to

$$
\eta\left(U(z)-y_{1}, p(z-v)\right)>\frac{1}{2} .
$$


The last two inequalities certainly will be satisfied for a choice of $y_{1}$ for which

$$
\eta\left(U(z)-p(z-v), y_{1}\right)>\frac{1}{2} \text { and } \eta\left(p(z+v)-U(x), y_{1}\right)>\frac{1}{2} .
$$

where $x, z \in Y$. To see the existence of $y_{1} \in Y$ satisfying (4), start by observing that for each $x, z \in Z$,

$$
\begin{gathered}
\eta(U(z)-p(z-x), p(u+x)-U(u)) \\
\geq \eta(U(z+u), p(z+u))>\frac{1}{2} .
\end{gathered}
$$

This inequality coupled with completeness of $Y$ implies that

$$
s=\sup \{U(z)-p(z-x): z \in Z\}
$$

and

$$
t=\inf \{p(u+x)-U(u): u \in Z\}
$$

exists in $Y$. and $\eta(s, t)>\frac{1}{2}$.

Now any $y \in Y$ satisfying

$$
\eta(s, y)>\frac{1}{2} \text { and } \eta(y, t)>\frac{1}{2},
$$

satisfies (4) and hence (3). The proof of the Theorem is now a routine application of Zorn's lemma.

Remark 7.20. For an analytic form of fuzzy Hahn-Banach theorem for normed spaces see Rhie and Hwang [80.

Remark 7.21. Results presented in this section are from Beg et al [6], [25], [26] and [27].

\section{Acknowledgement}

The author thanks Prof. Sorin Gal for a very careful reading and perceptive comments.

\section{References}

[1] S. Abian and A.B. Brown, A theorem on partially ordered sets, with applications to fixed point theorems, Canad. J. Math., 13 (1961), 78-82. 1

[2] C.D. Aliprantis and K.C. Border, Infinite Dimensional Analysis, Springer Verlag, Heidelberg - 1994. 1

[3] C.D. Alipantis, D.J. Brown and O. Burkinshaw, Edgeworth equilibria, Econometrica, 55, (1987), 1109-1137. 1

[4] I. Beg, $\sigma$-Complete fuzzy Riesz spaces, Resultate der Mathematik, 31 (1997), 292-299. 1

[5] I. Beg, Fixed points of fuzzy multivalued mappings with values in fuzzy ordered sets, J. Fuzzy Math., 6 (1998), 127-131. 1

[6] I. Beg, Extension of fuzzy positive linear operators, J. Fuzzy Math., 6(4)(1998), 849-855. 11, 1

[7] I. Beg, On fuzzy Zorn's lemma, Fuzzy Sets and Systems, 101(1) (1999), 181 - 183. 1

[8] I. Beg, Fixed points of fuzzy monotone maps, Archivum Math., (BRNO) 35(1999), 141 - 144. 1

[9] I. Beg, Fixed points of expansive mappings on fuzzy preordered sets, J. Fuzzy Math., 7(1999), 397 - 400. 1

[10] I. Beg, A general theorem on selectors of fuzzy multifunctions, J. Fuzzy Math., 9(1)(2001), 97-101. 1

[11] I. Beg, Extension of fuzzy Zorn's lemma to nontransitive fuzzy relations, J. Fuzzy Math., 10(3)(2002), 681-685.79. 1

[12] I. Beg, Fuzzy ordering and completeness of fuzzy metric spaces, J. Fuzzy Math., 10(4)(2002), 789-795. 1

[13] I. Beg, Some applications of fuzzy ordered relations, CUBO, 6(1)(2004), 103-114. 1

[14] I. Beg, Fuzzy multivalued functions, Bull. Allahabad Math. Soc., 21(2006), 41-104. 1

[15] I. Beg and S. Ashraf, Fuzzy equivalence relations, Kuwait J. Sci.and Eng., (35)(1A)(2008), 33-51. 1

[16] I. Beg and S. Ashraf, Fuzzy similarity and measure of similarity with Lukasiewicz implicator, New Math. and Natural Computation, 4(2)(2008), 191-206. 1

[17] I. Beg and S. Ashraf, Fuzzy dissimilarity and distance functions, Fuzzy Information and Engineering, 2 (2009), 205-217. 1

[18] I. Beg and S. Ashraf, Fuzzy inclusion and fuzzy similarity with Gödel implication operator, New Math. and Natural Computation, 5(3) (2009) 617-633. 1

[19] I. Beg and S. Ashraf, Similarity measures for fuzzy sets, Applied and Computational Math., 8(2) (2009), 192-202. 1

[20] I. Beg and S. Ashraf, Fuzzy Relations (ISBN 978-3-8383-2069-4), Lambert Academic Publisher, Germany 2009 1] 
[21] I. Beg and S. Ashraf, Fuzzy transitive relations, Fuzzy Systems and Math., 24(4) (2010), 162-169. 1

[22] I. Beg and S. Ashraf, Fuzzy set of inclusion under Kleene's implicator, Applied and Computational Math., 10(1)(2011), 65-77. 1

[23] I. Beg and S. Ashraf, Fuzzy relational calculus, Bulletin of the Malaysian Mathematical Sciences Society - to appear in 2013 [1]

[24] I. Beg and N. Butt, (Im)possibility theorems in fuzzy framework, Critical Review; a publication of Society for Mathematics of Uncertainity, IV(2010), 1-11. 1

[25] I. Beg and M. Islam, Fuzzy Riesz spaces, J. Fuzzy Math., 2(1) (1994), 211-229. 1, 1 , 1

[26] I. Beg and M. Islam, Fuzzy ordered linear spaces, J. Fuzzy Math., 3(3) (1995), 659-670. $1,1,1$

[27] I. Beg and M. Islam, Fuzzy Archimedean spaces, J. Fuzzy Math., 5(2)(1997),413-423. 1 , 1,1

[28] T.X. Bergstrom, Maximal elements of acyclic relations on compact sets, J. Economic Theory, 10 (1975), 403 - 404.1

[29] A. Billot, Economic Theory of Fuzzy Equilibria, Springer - Verlag, Berlin - 1992. 1

[30] G. Birkhoff, Lattice Theory, 3rd ed., Amer. Math. Soc. Colloq. Publ., Vol 25, AMS Providence, R.I. 1967. 1

[31] B.K. Bose and D. Sahani, Fuzzy mappings and fixed point theorems, Fuzzy Sets and Systems, 21(1987), 53-58. 1]

[32] G.J. Brown, A note on fuzzy sets, Inform. and Control, 18(1971), 32-39. 1 ]

[33] J. Caristi, Fixed point theorems for mapping satisfying inwardness conditions, Trans. Amer. Math. Soc., 215 (1976), 241-251. 1

[34] J. Caristi and W.A. Kirk, Geometric fixed point theory and inwardness conditions, Proceedings of the Conf. on Geometry of Metric and Linear Spaces, Lecture Notes in Mathematics 490, Springer Verlag, 1975. 1, 1

[35] E.W. Chapin, Jr. Set valued set theory, Part I, Notre Dame J. Formal Logic, XV(4) (1974), 619-634. 1

[36] E.W. Chapin, Jr. Set valued set theory, Part II, Notre Dame J. Formal Logic, XVI (2) (1975), 255-267. 1

[37] H.W. Corley, An existence results for maximizations with respect to cones, J. Optimization Theory and Appl., 34(1980), 277 - 281. 1

[38] H.W. Corley, Existence and Lagrangian duality for maximization of set-valued functions, J. Optimization Theory and Appl., 54(1987), 489 - 501. 1

[39] R. Cristescu, Ordered Vector Spaces and Linear Operators, Editura Academiei, Bucharest - 1976.

[40] A.C. Davis, A characterization of complete lattices, Pacific J. Math., 5(1955), 311-319. 1

[41] G. Debreu, Mathematical Economics: Twenty papers of Gerard Debreu, Cambridge University Press, Cambridge-1983. 1

[42] K.J. Devin, The Joy of Sets: Fundamentals of contemporary set theory, Springer-Verlag, New York - 1993. 1

[43] D. Dubois and H. Prade, Fundamentals of Fuzzy Sets, Kluwer Academic Publishers, Dordrecht - 2000. 1

[44] D. Dubois and H. Prade, On possibility theory, formal concept analysis and granulation, Applied and Computational Math., (2011), special issue, in press. 1

[45] I. Ekeland, On the variational principle, J. Math. Anal. Appl., 47(1974), 324-353. 1

[46] I. Ekeland, Nonconvex minimization problems, Bull. Amer. Math. Soc. (New Series), 1(1979), 443-474. 1

[47] K. Fan, A generalization of Tychonoff's fixed point theorem, Math. Ann., 142(1961), 305 - 310. 1

[48] J.X. Fang, On fixed point theorems in fuzzy metric spaces, Fuzzy Sets and Systems, 46(1992), 107-113. 1

[49] S.G. Gal, Approximate selection for fuzzy-set-valued mappings and applications, J. Fuzzy Math., 3(40(1995), 941-947.

[50] K. Goebel and W.A. Kirk, Topics in Metric Fixed Point Theory, Cambridge Stud. Adv. Math., 28, Cambridge University Press, London - 19901

[51] O. Hadzic, Fixed point theorems for multivalued mappings in some classes of fuzzy metric spaces, Fuzzy Sets and Systems, 29(1989), 115-125. 1

[52] P.R. Halmos, Naive Set Theory, Springer-Verlag, New York - 1974. 1

[53] P.J. He, The variational principle in fuzzy metric spaces and its applications, Fuzzy Sets and Systems 45(1992), 389-394. 11

[54] S. Heilpern, Fuzzy mappings and fixed point theorems, J. Math. Anal. Appl., 83(1981), 566-569. 1

[55] U. Hohle and L.N. Stout, Foundation of fuzzy sets, Fuzzy Sets and Systems 40(1991), 257-296. 1]

[56] P. Howard and J.E. Rubin, Consequences of The Axiom of Choice, American Mathematical Society, Providence, RI-1998. 11

[57] T. Jech, The Axiom of Choice, North Holland, Amsterdam - 1973. 1

[58] T. Jech, Set Theory, Academic Press, New York - 1978. 1

[59] J.S. Jung, C.Y. Cho and J.K. Kim, Minimization theorems for fixed point theorem in fuzzy metric spaces and applications, Fuzzy Sets and Systems, 61(1994), 199-207.

[60] J.S. Jung, Y.J. Cho, S.M. Kang and S.S. Chang, Coincidence theorems for set valued mappings and Ekeland's variational principle in fuzzy metric spaces, Fuzzy Sets and Systems, 79(1996), 239-250. 1

[61] O. Kaleva, A note on fixed points for fuzzy mappings, Fuzzy Sets and Systems, 15(1985), 99-100. 1 .

[62] O. Kaleva and S. Seikkala, On fuzzy metric spaces, Fuzzy Sets and Systems, 12(1984), 215-229. 1

[63] J.L. Kelley, General Topology, D.Von Nostrand Co., Inc., New York -1965. 1

[64] E.E. Kerre, Basic principles of fuzzy set theory for the representation and manipulation of imprecision and uncertainty. In: Introduction to the Basic Principles of Fuzzy Set Theory and some of its Applications, E. E. Kerre, ed., Communication and Cognition, Gent, second revised edition (1993), 1-158. 1

[65] E.E. Kerre, The impact of fuzzy set theory on contemporary mathematics, Applied and Computational Math., (2011), special issue, in press. 1 
[66] W.A. Kirk, Caristi's fixed point theorem and metric convexity, Colloq. Math., XXXVI (1976), 81-86. 1, 1

[67] B. Knaster, Un theoreme sur les fonctions d'ensembles, Ann. Soc. Polon. Math., 6(1928), 133-134. 1 .

[68] S. Kundu, Similarity relations, fuzzy linear orders and fuzzy partial orders, Fuzzy Sets and Systems, 109 (2000), 419 - 428. 1

[69] B.S. Lee and S.J. Cho, A fixed point theorem for contractive-type fuzzy mappings, Fuzzy Sets and Systems, 61(1994), 309-312. 1

[70] H.X. Li and V.C. Yen, Fuzzy Sets and Fuzzy Decision-Making, CRC Press. Inc., London - 1995.1

[71] J. Lukasiewicz, A numerical interpretation of the theory of propositions, Ruch Filozoficzny 23(1922), 92-94. 1

[72] J.M. Merigo and A.M. Gil-Lafuente, An overview of fuzzy research in the ISI web of knowledge, Proc. World Congress on Engineering Vol. I , London-2009 (ISBN: 978-988-17012-5-1). 1 ]

[73] J.C. Moore, Zermelo's axiom of choice: Its origins, development, and influence, Springer-Verlag, New York-1982. 1

[74] C.V. Negoita and D. A. Ralescu, Applications of fuzzy sets to system analysis, Fuzzy Sets and Systems, 1(1978), $155-167$. 1

[75] V. Novak, Fuzzy Sets and Their Applications, Adam Hilger, Bristol, England - 1989. 1

[76] S.A. Orlovsky, Decision making with a fuzzy preference relation, Fuzzy Sets and Systems, 1(1978), 155 - 167.1

[77] J.Y. Park and J. U. Jeong, Common fixed points of fuzzy mappings, Fuzzy Sets and Systems, 59(1993), 231-235. 1.

[78] N. Prati, On the comparison between fuzzy set axiomatizations, Fuzzy Sets and Systems, 46(1992), 167-175. 1

[79] N. Prati, On the notion of fuzzy set, Stochastica XIII-1(1992), 101-114. 1

[80] G.S. Rhie and I. A. Hwang, On the fuzzy Hahn-Banach theorem- an analytic form, Fuzzy Sets and Systems 108(1999), 117-121. 1

[81] M.K. Richter, Revealed preference theory, Econometrica 34(1966), 635-645. 1

[82] H. Rubin and J.E. Rubin, Equivalence of the Axiom of Choice, North Holland, Amsterdam -1963. 1

[83] B. Russell, Vagueness, Australian J. Phil., 1(1)(1923), 84-92 1

[84] R.E. Smithson, Fixed points of order preserving multifunctions, Proc. Amer. Math. Soc., 28(1) (1971), 304-310. 1

[85] E. Szpilrajn, Sur l'extension de l'ordre partiel, Fundamenta Mathematicae, 16(1930), 386-389. 1

[86] A. Tarski, A lattice theoretic fixed point theorem and its applications, Pacific J. Math., 5(1955), 285-309. 1

[87] P. Venugopalan, Fuzzy ordered sets, Fuzzy Sets and Systems, 46(1992), 221-226. 1] 1

[88] P. Vijayaraju and M. Marudai, Fixed point for pair of maps in fuzzy metric spaces, J. Fuzzy Math., 8(1)(2000), 117-122. 1]

[89] A.C. Zaanen, Introduction to Operator Theory in Riesz Spaces, Springer-Verlag, Berlin - 1997.

[90] L.A. Zadeh, Fuzzy Sets, Inform. and Control, 8(1965), 338-353. 1] 1 ]

[91] L.A. Zadeh, Similarity relations and fuzzy orderings, Inform. Sciences, 3(1971), 177-200. 1, 1

[92] E. Zeidler, Nonlinear Functional Analysis I: Fixed Point Theory, Springer Verlag, New York - 1986. 1

[93] J. Zhou and G. Tian, Transfer methods in characterizing the existence of maximal elements for binary relations on compact or noncompact sets, SIAM J. on Optimization, 2(1992), 360 - 375. 1

[94] H.J. Zimmermann, Fuzzy Set Theory and its Applications, Kluwer Academic Publishers, Dorderecht - 1991.1

[95] M. Zorn, A remark on method in transfinite algebra, Bull. Amer. Math. Soc., 41(1935), 667 - 670.

1 